\title{
Rewarding Trading Skills Without Inducing Gambling
}

\author{
IGOR MAKAROV and GUILLAUME PLANTIN*
}

\begin{abstract}
This paper develops a model of active asset management in which fund managers may forego alpha-generating strategies, preferring instead to make negative-alpha trades that enable them temporarily to manipulate investors' perceptions of their skills. We show that such trades are optimally generated by taking on hidden-tail risk, and that they are more likely to occur when fund managers are impatient, and when their trading skills are scalable and generate a high profit per unit of risk. We propose long-term contracts that deter this behavior by dynamically adjusting the dates on which the manager is compensated in response to her cumulative performance.
\end{abstract}

\footnotetext{
* Makarov is with the London School of Economics and Plantin is with the Toulouse School of Economics. We are most grateful for helpful comments made by the editor (Cam Harvey) and two anonymous referees, and the following: Gilles Chemla, Zhiguo He, Christopher Hennessy, Roni Kisin, Peter Kondor, Semyon Malamud, Gustavo Manso, Andrea Prat, Jan Schneider, Lars Stole, Ilya Strebulaev, and seminar participants at Chicago Booth, Imperial College, Kellogg, LBS, Pompeu Fabra, Rochester, Queen Mary, University of London, University of Lugano, University of Tokyo, Vienna University, American Finance Association 2011 Denver meetings, HKUST Finance Symposium, Second Paris Hedge Fund Conference, Third Annual Paul Woolley Centre Conference, Society for Economic Dynamics 2010 Montreal Meeting, Second Theory Workshop on Corporate Finance and Financial Markets, New York, and UBC Summer Conference 2010. Plantin benefitted from a European Research Council Starting Grant ( $\mathrm{N}^{\circ} 263673$ - RIFIFI)
} 
The last thirty years have witnessed two important developments in financial markets. First, a rapid pace of financial innovation has made it possible to slice and combine a large variety of risks by trading a rich set of financial instruments. Second, the management of large amounts of capital has been delegated to entities such as hedge funds and bank proprietary desks that are neither subject to significant trading restrictions nor required to disclose publicly the details of their positions.

The amount of capital available to such entities crucially depends on investors' perceptions of their "alpha" - that is, their ability to generate excess returns above the level of fair compensation for risk. Combined with the relative opaqueness of these entities and their vast risk-taking opportunities, this creates room for a particular type of agency problem.

Fund managers who are running out of alpha-generating strategies may find it tempting to pretend otherwise, and to take risky positions with zero or even negative alpha that may temporarily improve their perceived reputation in case of favorable outcomes. Strategies that generate frequent small positive excess returns that are offset by very rare and large losses seem especially well suited to disguising luck as skill. As Rajan (2008) puts it: "How can untalented investment managers justify their pay? Unfortunately, all too often it is by creating fake alpha - appearing to create excess returns but actually taking on hidden tail risk." Consistent with this view, Jiang and Kelly (2012) showed that a significant number of hedge funds are indeed exposed to tail risk.

Creating fake alpha by taking on hidden tail risk does not seem to be limited to the hedge fund industry. For example, in its 2008 report to shareholders intended to analyze the causes of its subprime losses, UBS concluded that "The UBS compensation and incentivization structure did not effectively differentiate between the creation of alpha versus the creation of return based on a low cost of funding." More systematically, Acharya et al. (2010) argued that the manufacture of tail risk through deliberate retention of senior tranches on poor collateral by U.S. banks was an important ingredient 
of the 2008 banking crisis.

The perverse incentives to enter into (at best) zero-alpha gambles come with a number of costs. First, they defeat the purpose of delegated asset management, which is meant to combine "brains and resources" optimally in order to achieve superior returns. Second, they lead to a misallocation of capital. Finally, the manufacture of tail risk has far-reaching consequences for overall financial stability, and for the taxpayer when gambling institutions benefit from public safety nets, either by law or because they are systemically important.

The goal of this paper is to develop a new framework for the study of these risktaking incentives. We study those situations where managers find it optimal to fake their alpha and propose a new class of contracts that eliminate any incentives to employ such strategies.

Our model builds upon the frictionless benchmark of Berk and Green (2004), who studied career concerns in delegated fund management. In their model, a fund manager and investors discover the manager's alpha-generating skills by observing her realized returns. The excess returns that a manager is expected to generate increase with respect to her skills, but decrease as she gains more funds under management. Competitive investors supply funds to the manager until they earn a zero net (after fees) expected return. At the beginning of each period, the manager sets fees that enable her to reach the optimal fund size, and extract the entirety of the surplus that she generates. Learning and competition among investors imply that both fund flows and managerial compensation strongly depend on the manager's record.

We add one particular friction to this model. We suppose that the manager may secretly enter into zero-alpha trades with the sole purpose of manipulating investors' perceptions of her skills. In what follows, we refer to this opportunistic behavior as inefficient risk shifting or gambling. In contrast to many earlier papers on risk shifting, we propose a general setting in which the fund manager can secretly choose to take on positions with any arbitrary payoff distributions. This captures the large set of trading 
opportunities available to modern managers, and is therefore an important case to consider.

We first study the impact of this friction in the case where the manager and investors sign only short-term contracts. Three factors that are conducive to inefficient risk shifting emerge from our analysis. The first is the size of the alpha per unit of risk that can be generated by a skilled manager. If it is large, the history of returns has a large impact on investors' beliefs about the manager's ability to generate future excess returns. The second factor is the scalability of trading skills - that is, the sensitivity of expected excess returns to fund size. If trading skills are scalable, a good reputation translates into a large future fund size and thus into large future profits. Finally, because the manager can manipulate her reputation only temporarily, she finds it more valuable to do so when she is more impatient. These three factors determine the convexity of future expected gains as a function of realized returns, and thus affect inefficient risk-shifting incentives. In particular, the model predicts that "fallen-star" managers (those who show high initial potential but who realize disappointing returns) are particularly prone to gambling. For a calibration consistent with that of Berk and Green (2004), we find that their efficient equilibrium with short-term contracts breaks down, in the sense that any equilibrium must involve some degree of risk shifting.

We are able fully to characterize such equilibria with risk shifting in a simplified version of the model, where the manager maximizes a combination of her expected current return and the expected reputation that results from it. Interestingly, even though we impose no restriction on the risk profiles available to the manager, we show that she finds it optimal to manufacture hidden-tail risk. In other words, she sells disaster insurance, and adds some noisy payoff to the collected premium so that investors cannot discover the exact nature of the trade.

We then consider long-term contracts; and here we follow two distinct lines of inquiry. First, we consider a contract popular in the hedge fund industry, in which the manager's profits are given by a performance fee above a high-water mark. Similar to 
Panageas and Westerfield (2009), we find that without new inflows/outflows triggered by realized performance, the performance fee with high-water mark is not conducive to inefficient risk shifting. In the presence of fund flows, however, we show that the high-water mark contract does not generally solve the risk-shifting problem.

Second, we exhibit an optimal contract that fully eliminates risk-shifting incentives. The contract is designed to discriminate between skills and luck. It exploits the fact that the impact of gambling on investors' beliefs vanishes in the long-run, when true skills are eventually revealed. The contract consists in deferring payments to the manager at dates that vary depending on her cumulative performance. The promised payment also evolves in order that it always at least matches the managers outside options, thereby leading her not to renegotiate the contract. As we detail in Section 3, this mechanism is highly reminiscent of the recent proposals for bankers' compensation reforms issued both by public authorities and the industry itself. All such proposals consist of a deferral of bonuses together with a clawback mechanism ensuring that the initial promised payment is revised with the benefit of hindsight. We offer theoretical foundations for these proposals. More importantly, we qualify them, suggesting in particular that it is important to adjust the timing of the compensation dynamically, notably by further postponing it upon observing a poor track record. In our model, committing to pay a bonus at a fixed date may generate inefficient gambling in general, even if this date is remote and even if the bonus is adjusted via a clawback provision. The dynamic revision of the payment date turns out to be important, and yet it is absent from the current suite of proposed reforms.

To our knowledge this paper is the first to derive a formal connection between fund managers' career concerns and alpha-faking through hidden-tail risk in a fully rational environment. More precisely, we bridge two strands of literature, namely that on risk shifting and that on career concerns. The risk-shifting friction was first introduced by Jensen and Meckling (1976) as a source of value destruction within overly leveraged firms. Arguably, this friction is particularly relevant in the context of sophisticated 
financial institutions that can swiftly alter their risk profiles. Accordingly, there is a large amount of literature on asset pricing that considers the impact of nonconcave objective functions on the risk-shifting incentives of fund managers who have access to dynamically complete markets. Contributions include those of Basak, Pavlova, and Shapiro (2007), Carpenter (2000), and Ross (2004). In common with them, we seek to identify the risk-taking strategies that optimally respond to nonconcave objectives. We extend this line of research in two directions. First, nonconcavities in the manager's objective are not assumed in our model. Rather, they arise endogenously from reputational concerns in a truly dynamic environment. Second, we exhibit optimal contracts that eliminate the costs of this friction.

Acharya, Pagano, and Volpin (2012) also developed a model in which career concerns may lead managers to destroy value, but by a different means from that of manipulating a payoff distribution. As in our model, limited commitment prevents managers from receiving insurance against the risk that their reputation deteriorates. It is assumed that learning about managerial skills can take place only if managers run the same project for sufficiently long. Thus risk-averse managers may prefer to churn across projects in order to prevent learning. Whilst this shields them from reputational risk, it inefficiently slows down the identification of good managers. Malliaris and Yan (2012) considered a two-period model in which a manager may be tempted to take on tail risk in order to manipulate her expected reputation. Their setup is related to the static version of our model, which we solve for equilibria with risk shifting. In our case the main difference is that we do not impose binary payoffs, as they do.

Our paper is also related to that of Goetzmann et al. (2007), who studied manipulationproof measures of managerial performance. They showed that to be manipulation-proof a measure should take the form of a concave utility function averaged over the return history. We also show that if the fund manager has a nonconcave continuation utility she can engage in inefficient risk shifting, and that optimal contracts are aimed at concavifying the manager's objective . 
Finally, our study relates to two recent extensions of the Berk and Green (2004) model. First, Berk and Stanton (2007) applied the Berk and Green setup to closed-end funds. In this case, learning affects the net asset value of the fund and not its size, which is fixed by construction. Berk and Stanton showed that the impact of learning explains several characteristics of the closed-end fund discount, and that the behavior of this discount crucially depends on the nature of the compensation contract. Second, Dang, Wu, and Zechner (2008) studied an extension of Berk and Green's approach in which a management company can fire a manager if her performance is not good enough. They restricted their analysis to short-term compensation contracts and solved for the optimal firing rule.

The remainder of our paper is organized as follows. Section I develops our baseline model of career concerns, and studies the impact of the risk-shifting friction when the manager and investors sign only short-term contracts. Section II studies long-term contracts. Section III concludes. Technical proofs are relegated to the appendix.

\section{Career Concerns and Inefficient Risk Shifting}

In Section $A$, we introduce and solve a frictionless model of career concerns in delegated asset management that closely follows the approach used by Berk and Green (2004). In Section $B$, we introduce a risk-shifting friction to this benchmark approach, and study its impact on the equilibrium in the presence of short-term contracts. In Section $C$, we fully characterize equilibria with risk shifting in a simplified version of our baseline model. In Section $D$, we discuss the costs of risk-shifting strategies.

\section{A. Frictionless Benchmark: The Berk and Green model}

Time is discrete and is indexed by $\{n \Delta t\}$, where $n \in \mathbb{N}$ and $\Delta t>0$. There is a single consumption good which serves as the numéraire. Agents are of two types: a manager and investors. Agents live forever, are risk-neutral, and discount future 
consumption at the instantaneous rate $r>0$.

The manager is protected by limited liability and is thus unable to have negative consumption. Investors receive a large endowment of the consumption good at each date $n \Delta t$, the manager does not. The manager has exclusive access to an investment technology. If the manager invests $q_{t}$ consumption units at date $t$ using her technology, she generates $q_{t+\Delta t}$ units at date $t+\Delta t$ such that

$$
q_{t+\Delta t}=q_{t} e^{\left(r+a \theta-c\left(q_{t}\right)-\frac{\sigma^{2}}{2}\right) \Delta t+\sigma\left(B_{t+\Delta t}-B_{t}\right)}
$$

where $\left(B_{t}\right)_{t \geq 0}$ is a standard Wiener process, and $a$ and $\sigma$ are strictly positive numbers.

The parameter $\theta \in\{0,1\}$ measures the manager's skills. It is unobservable by both the manager and investors. All other parameters are common knowledge. The parameter $a$ is the alpha that a skilled manager can generate with her first dollar. As in Berk and Green (2004), the cost $c\left(q_{t}\right)$ captures the fact that many arbitrage opportunities or informational rents in financial markets are not perfectly scalable. The function $c$ is increasing, taking the form

$$
c(q)=\beta q^{\frac{1}{\alpha-1}}
$$

where $\alpha \geq 1, \beta>0$.

All agents share the common date- 0 prior to which the manager is endowed with high skills - that is, that $\theta=1$ - with probability $\pi_{0} \in(0,1)$. Except for the manager's skills, each action and the manager's realized returns are publicly observable at each date $n \Delta t$. Thus, information is symmetric across agents.

Let $\pi_{t}$ denote the probability that the agents assign to the possibility that the manager is skilled at a given date $t$. We will refer to $\pi_{t}$ as the manager's perceived skills. Given $\pi_{t}=\pi$, the net expected surplus created over $[t, t+\Delta t]$ if the manager invests $q$ units is

$$
q\left(\pi e^{(a-c(q)) \Delta t}+(1-\pi) e^{-c(q) \Delta t}-1\right)
$$


which in the limit as $\Delta t \rightarrow 0$ becomes $q(a \pi-c(q))$. Thus the manager creates the maximal net expected surplus over $[t, t+\Delta t]$ if she invests $q(\pi)$ that solves

$$
q(\pi)=\arg \max _{q} q\left(\pi e^{(a-c(q)) \Delta t}+(1-\pi) e^{-c(q) \Delta t}-1\right)
$$

We will refer to such $q(\pi)$ as the optimal fund size. Denoting $v(\pi) \Delta t$ as the maximal expected profit over $[t, t+\Delta t]$ that corresponds to this optimal fund size, we have:

\section{LEMMA 1:}

$$
\begin{aligned}
\lim _{\Delta t \rightarrow 0} q(\pi) & =\left(\frac{\alpha-1}{\alpha \beta} a \pi\right)^{\alpha-1} \\
\lim _{\Delta t \rightarrow 0} v(\pi) & =\beta^{1-\alpha} \frac{(\alpha-1)^{\alpha-1}}{\alpha^{\alpha}}(a \pi)^{\alpha} .
\end{aligned}
$$

Proof of Lemma 1: See Appendix.

Using a power specification (2) for the cost function $c(q)$, the expected instantaneous surplus $v(\pi)$ is proportional to $\pi^{\alpha}$ as $\Delta t$ becomes small. The parameter $\alpha$ captures the scalability of the trading skills. As $\alpha$ increases, the manager's skills $\pi$ become more scalable and therefore, her expected profit becomes more sensitive to her reputation, in other words, it becomes more convex in $\pi$. In the hedge fund universe, global macro strategies are typically quite scalable. At the same time, strategies based on shareholder activism may be more difficult to spread over increasing amounts of capital.

Berk and Green specified a linear cost function $c$ corresponding to $\alpha=2$. They showed that their model matches quantitatively well the empirically observed relationship between realized returns of mutual funds and inflows/outflows. In the remainder of the paper, we will generally restrict the analysis to a somewhat simpler limiting case in which $\alpha=1$. In this case, the cost is zero for $q<1$ and infinite for $q>1$. Thus, the manager can scale up the fund at no cost up to an upper bound that is (without loss of generality) normalized to 1 . The optimal fund size is thus always 1 and $v(\pi)$ is linear in $\pi$. In other words, in this case perceived skills affect the expected rate of 
return of the fund but the optimal fund size remains constant, normalized to 1.

To realize any surplus, investors must delegate their funds to the manager and agree with her on the profit-sharing rule. We assume that investors are competitive and can fully commit to a contract. At the same time, at the end of any period, the fund manager is free to walk away from a contract and sign a new one with competing investors. More precisely, at the end of each period $[t, t+\Delta t]$, after returns are realized and all contractual transfers for the period are made, the fund manager is free to terminate a contract without financial obligation to its investors, and to enter a new one with new investors starting at period $[t+\Delta t, t+2 \Delta t]$. In other words, commitment is one-sided. This is a common assumption in labor economics. We find it to be all the more plausible in the financial services industry where fund managers can swiftly move between jobs and financial centres because their activity entails making few specific investments. Limited cross-border enforcement precludes covenants that would make such moves costly.

We first study incentives to take risk when investors and the manager cannot enter into long-term contracts, but rather simply interact in a spot labor market at each date. Our goal is to determine whether incentives created by market forces alone can discipline managers who are concerned about their reputations in the labor market

We therefore postpone the analysis of explicit long-term contracts until Section II, and instead assume, like Berk and Green, that the manager enters only into one-period contracts with investors:

ASSUMPTION 1: At each date $t$, the manager offers investors a one-period asset management contract.

Assumption 1 only imposes a restriction on the horizon of contracts: The parties cannot contract at date $t$ on actions or transfers beyond date $t+\Delta t$. Parties are free to write any one-period contracts, however, subject only to the limited-liability constraint of the manager. We next show that this restriction to short-term contracts does not lead 
to any misallocation of capital in the absence of frictions.

LEMMA 2: Under Assumption 1, the manager adopts the optimal fund size $q(\pi)$ at each date and extracts the maximal expected surplus $v(\pi) \Delta t$.

Proof of Lemma 2: One only needs to exhibit a particular contractual arrangement that enables the manager to raise $q(\pi)$ at each period, and to receive an expected compensation $v(\pi) \Delta t$ over $[t, t+\Delta t]$. In the absence of any frictions, there are many different arrangements in which this can be achieved. For example, the manager can simply ask investors at the beginning of each period to pay her a salary $v(\pi) \Delta t$, collect funds $q(\pi)$ from them, invest, and leave them the date- $(t+\Delta t)$ proceeds. She may alternatively, as assumed by Berk and Green (2004), quote a fee $f \Delta t$ at the beginning of each period. The fee is the fraction of the date- $(t+\Delta t)$ assets under management (before any new inflows/outflows of funds) that accrues to the manager. If a manager with perceived skills $\pi$ quotes a fee $f \Delta t$, competitive investors will supply funds as long as their net expected rate of return is equal to $r$. Thus, their fund supply $q(f)$ solves

$$
(1-f \Delta t)\left(\pi e^{(r+a-c(q(f))) \Delta t}+(1-\pi) e^{(r-c(q(f))) \Delta t}\right)=e^{r \Delta t}
$$

Therefore as $\Delta t \rightarrow 0, q(f)$ solves

$$
\pi a=c(q(f))+f
$$

The manager maximizes her expected profits by choosing $f$ such that:

$$
f=\arg \max _{f} f \Delta t \times q(f)=\arg \max _{f}(\pi a-c(q(f))) \Delta t \times q(f),
$$

which implies that the manager collects the maximal expected surplus $v(\pi) \Delta t$.

Q.E.D.

This baseline model is essentially identical to that of Berk and Green (2004). The 
main modeling difference is that the distribution of skills is binomial in our setup while it is Gaussian in theirs. With our specification, the model is stationary in perceived skills $\pi$, and is therefore more tractable. In particular, this specification yields a tractable formulation of the manager's total expected profit from date 0 onwards. While this is not particularly useful in the frictionless environment of Berk and Green, it turns out to be instrumental when we introduce asymmetric information between the manager and investors.

Let $\left(\pi_{n \Delta t}\right)_{n \geq 0}$ denote the process that describes the manager's perceived skills at each date. From Lemma 2, the manager's continuation utility is

$$
V(\pi, \Delta t)=E\left[\sum_{n=0}^{\infty} e^{-r n \Delta t} v\left(\pi_{n \Delta t}\right) \Delta t \mid \pi_{0}=\pi\right]
$$

where $v(\pi)$ is given by (4). The following proposition shows that this continuation utility converges to a simple limit when $\Delta t$ becomes small.

PROPOSITION 1: Let

$$
V(\pi)=\lim _{\Delta t \rightarrow 0} V(\pi, \Delta t) .
$$

We have

$$
V(\pi)=\int_{0}^{1} G(\pi, x) v(x) d x,
$$

where

$$
G(\pi, x)=\frac{2 \sigma^{2}}{\psi a^{2} x^{2}(1-x)^{2}}\left\{\begin{array}{lll}
g(1-\pi) g(x) & \text { if } & 0 \leq x \leq \pi \\
g(\pi) g(1-x) & \text { if } & \pi \leq x \leq 1
\end{array}\right.
$$

and

$$
\begin{aligned}
\psi & =\sqrt{1+\frac{8 r \sigma^{2}}{a^{2}}}, \\
g(u) & =u^{\frac{1}{2}+\frac{1}{2} \psi}(1-u)^{\frac{1}{2}-\frac{1}{2} \psi} .
\end{aligned}
$$

Convergence of $V(\pi, \Delta t)$ to $V(\pi)$ when $\Delta t \rightarrow 0$ is uniform over $\pi \in(0,1)$. 


\section{Proof of Proposition 1: See Appendix.}

In the remainder of the paper, all results will be established for $\Delta t$ that is sufficiently small for us to approximate the manager's continuation utility with its continuous-time limit (6).

Expression (6) can be interpreted as an expectation over the instantaneous surplus $v(x)$ weighted by a discount factor $G(\pi, x)$. The factor $G(\pi, x)$ has an intuitive interpretation: it measures the discounted frequency of the future dates at which the manager will have a perceived ability $x$ given that she starts out with perceived skills $\pi$. Notice that the factor $G$ depends only on $a / \sigma$, which governs the speed at which agents learn about the manager's skills, and on the discount rate $r$. The cost parameters $\alpha$ and $\beta$ affect only the instantaneous profit $v$ from (4). This is because the cost is known and thus filtered out by the agents when inferring skills from realized returns. It is easy to verify that

$$
\int_{0}^{1} G(\pi, x) x d x=\frac{\pi}{r} .
$$

Thus, in the case $\alpha=1$, the continuation utility $V(\pi)$ is proportional to $\pi$.

We now depart from this frictionless benchmark set by Berk and Green, and intro-

duce informational asymmetry between the manager and investors. We posit that the manager may secretly enter into zero-alpha trades in order to manipulate her perceived skills temporarily as opposed to efficiently investing in the alpha-generating technology described in (1). We will show that the spot labor market interactions considered thus far may generate perverse incentives in the presence of this friction.

\section{B. Risk Shifting}

The Berk and Green model assumes a frictionless interaction between the manager and investors. While this is a very useful benchmark, in reality several informational frictions may affect the delegation of asset management to sophisticated entities such as hedge funds and investment banks. As the following quote from the Financial Stability 
Forum "Principles for Sound Compensation Practices" suggests, the impossibility of perfectly monitoring shifts in risk exposures within such institutions can be particularly problematic:

In principle, if risk management and control systems were strong and highly effective, the risk-taking incentives provided by compensation systems would not matter because risk would stay within the firm's appetite. In practice, all risk management and control systems have limitations and, as the current crisis has shown, they can fail to properly control risks. The incentives provided by compensation can be extremely powerful. Without attention to the risk implications of the compensation system, risk management and control systems can be overwhelmed, evaded, or captured by risk-takers.

To study such incentives for surreptitiously shifting risk exposures, we introduce the following friction to the baseline model:

ASSUMPTION 2: (Risk-shifting technology). At each date, the manager can secretly invest all or part of her funds in an alternative technology whose returns are perfectly scalable and independent of the returns on the technology described in (1). This technology enables her to generate a one-period gross return with any arbitrary distribution over $[0, \infty)$ with mean $e^{r^{\prime} \Delta t}$, where $r^{\prime} \leq r$. Investors observe returns realized at the reporting and contracting dates $n \Delta t$, at which the manager's position is marked-tomarket.

We will now briefly comment on our modeling choice. We interpret the alphagenerating technology (1) available to the manager as the investment strategy that she agrees with the investors and/or her supervisors. In practice this strategy may vary over time, and involve shifts in asset selection, asset allocation, or overall risk exposure. But such shifts are agreed between the parties. For simplicity, we abstract 
from them and, following Berk and Green, we model this agreed trading strategy as a simple production function (1).

The "risk-shifting" technology that the manager may secretly use reflects the positions that she can conceal from the investors and /or her supervisors by evading the various control and risk management systems put in place to monitor her. To bring our results into sharper focus, we assume that these trades are detrimental to investors in that they do not generate a positive risk-adjusted expected excess return. Moreover, we allow for the possibility that concealing these trades from investors comes at a cost $r-r^{\prime}$. The assumption that these trades are independent of the alpha-generating technology ensures that they cannot be used for arbitrage purposes. Because such trades yield less in terms of expectation than the manager's alpha-generating technology (1), she will not invest her own funds in them. In the presence of career concerns, however, the manager may be tempted to use the "risk-shifting" technology because it provides her with an opportunity to manipulate investors' beliefs about her reputation.

Given the large set of trading opportunities available to sophisticated managers, we consider a general setting in which the fund manager can secretly choose any arbitrary payoff distribution. One of the questions we are interested in relates to understanding which trades work best in manipulating investors' beliefs. For expositional simplicity, we assume the perfect scalability of these trades. It is straightforward matter to extend our analysis to the case in which large trades are more difficult to hide than small trades.

The manager in our model may literally be interpreted as an individual trader, or may alternatively be a desk or a division that collectively decides to breach its mandate or exceed its risk limits. In the remainder of the paper, we refer to the secret use of this inefficient technology by the manager, as opposed to the transparent risk taking induced by the alpha-generating technology (1), as "risk shifting" or "gambling".

We now study whether the friction introduced in Assumption 2 affects the outcomes in the Berk and Green environment for short-term contracts (Assumption 1). The first type of opportunistic behavior could occur if the manager secretly invests a portion of 
her funds at the risk-free rate $r^{\prime}$, rather than using her alpha-generating technology. This represents the case where a manager performs well from January to November, say, and then prefers to stop investing actively in order to lock in a profit. We then have the following result.

PROPOSITION 2: If

$$
r^{\prime} \leq r-\sigma^{2} / 2
$$

then the manager does not secretly invest at the risk-free rate.

Proof of Proposition 2: See Appendix.

The remainder of the paper focuses on situations in which the manager uses the risk-shifting technology to increase rather than reduce risk. We believe that this is a more important question because such excessive risk taking could contribute to financial instability, as suggested by the recent financial crisis . We therefore assume in what follows that condition (10) holds.

We now characterize the manager's incentives to gamble secretly (and inefficiently). Suppose that the economy is in an equilibrium in which investors believe that the manager always invests in the alpha-generating technology (1). We assess whether the manager could be tempted to deviate and enter into a one-shot gamble.

Suppose therefore that the manager gambles during her first trading round, realizes a return $R$, and from then on does not gamble. Suppose that investors believe instead that she has never gambled. Let $\pi_{n \Delta t}$ be the manager's (correct) belief about her skills at date $n \Delta t$ and let $\pi_{n \Delta t}^{R}$ be investors' (incorrect) perception of her skills at date $n \Delta t$. We have

PROPOSITION 3:

$$
\pi_{t}^{R} \equiv \lim _{\Delta t \rightarrow 0, n \Delta t \rightarrow t} \pi_{n \Delta t}^{R}=\frac{\pi_{t} R^{\frac{a}{\sigma^{2}}}}{1-\pi_{t}+\pi_{t} R^{\frac{a}{\sigma^{2}}}} .
$$

Proof of Proposition 3: See Appendix. 
Remark. In principle, a realization of $R=0$ should perfectly reveal to investors that the manager gambled because her alpha-generating technology delivers strictly positive returns with a probability of one. We assume instead that $\pi_{t}^{R}$ is continuous at zero, so that investors infer $\pi=0$ from observing $R=0$. Equivalently, we could assume that traders who are caught gambling are excluded from the market.

The continuation utility of the manager after the return $R$ is realized is

$$
W(\pi, R, \Delta t)=E\left[\sum_{n=0}^{\infty} e^{-r n \Delta t} v\left(\pi_{n \Delta t}^{R}\right) \Delta t \mid \pi_{0}=\pi\right]
$$

For $\Delta t$ small, (6) and (11) imply that:

$$
W(\pi, R) \equiv \lim _{\Delta t \rightarrow 0} W(\pi, R, \Delta t)=\int_{0}^{1} G(\pi, x) v\left(\frac{x R^{\frac{a}{\sigma^{2}}}}{1-x+x R^{\frac{a}{\sigma^{2}}}}\right) d x
$$

Notice that as $\Delta t \rightarrow 0$ the mean of the gamble, $e^{r^{\prime} \Delta t}$, converges to one irrespective of $r^{\prime}$. The manager therefore chooses a unit mean gamble whose distribution maximizes her expectation over $W(\pi, R)$. Formally, denoting $M$ the set of Borelian probability measures over $[0,+\infty)$, she solves

$$
\begin{aligned}
& \max _{\mu \in M} \int_{0}^{\infty} W(\pi, R) d \mu(R) \\
& \text { s.t. } \quad \int_{0}^{\infty} R d \mu(R)=1 .
\end{aligned}
$$

In the electronic appendix, we show that the generic solution to (13) is attained using a binary gamble - or a measure comprised of two atoms, one above 1 and the other below. If these two atoms coincide at 1 , the manager does not find it worthwhile to gamble, and an equilibrium without inefficient risk shifting can be sustained. If they do not, then the optimal binary gamble is not degenerate, which means that such an equilibrium without risk shifting does not exist. 
Obviously, a sufficient condition that makes gambling undesirable is that $W(\pi, R)$ is concave in $R$. More generally, the optimal gamble is determined by the convexity properties of $W(\pi, R)$, which in turn depend on the parameters $a, \sigma, \alpha$, and $r$. We have the following result.

PROPOSITION 4: Suppose that Assumptions 1 and 2 hold.

i) If

$$
\frac{a}{\sigma^{2}} \leq \frac{1}{\alpha}
$$

then there exists an equilibrium in which the manager extracts the entire expected surplus and does not engage in risk shifting.

ii) If

$$
\frac{a}{\sigma^{2}}>\frac{1}{\alpha} \quad \text { and } \quad r>\frac{\alpha(\alpha-1) a^{2}}{2 \sigma^{2}}
$$

then such an equilibrium does not exist.

Proof of Proposition 4: See Appendix.

To gain a better understanding of the results in Proposition 4, suppose that the manager tries to "pick up nickels in front of a steamroller", in other words, she gambles and realizes an instantaneous return of $1+\varepsilon$ with probability $1 /(1+\varepsilon)$, where $\varepsilon$ is small, or loses everything. Then from (11), in case of success, her new reputation is

$$
\pi^{R}=\frac{\pi(1+\varepsilon)^{\frac{a}{\sigma^{2}}}}{1-\pi+\pi(1+\varepsilon)^{\frac{a}{\sigma^{2}}}},
$$

which for $\pi$ and $\varepsilon$ small enough is approximately

$$
\pi^{R} \simeq \pi_{0}\left(1+\frac{a}{\sigma^{2}} \varepsilon\right)
$$

Further, Lemma 6 in the Appendix shows that if $r$ is not too small, then the continuation utility of the manager $V(\pi)$ behaves as $\pi^{\alpha}$ as $\pi \rightarrow 0$. Therefore, the manager's 
net expected gain from the gamble for $\pi$ and $\varepsilon$ small enough is proportional to

$$
\frac{1}{1+\varepsilon}\left(\pi^{R}\right)^{\alpha}-\pi^{\alpha} \simeq \pi^{\alpha}\left(\frac{\alpha a}{\sigma^{2}}-1\right) \varepsilon
$$

Thus, whether there is risk shifting or not depends on whether the ratio $\alpha a / \sigma^{2}$ is greater or less than 1 .

Proposition 4 shows that risk shifting is particularly appealing when three conditions are met. First, managerial skills generate a high alpha per unit of risk $\left(a / \sigma^{2}\right.$ is large). One can see from (16) that in this case, realized returns have a large impact on investors' beliefs. Second, the manager's skills are sufficiently scalable ( $\alpha$ is large). In this case, positive news about her skills translates into a large increase in expected future fund size, and thus into large future expected profits. Finally, the manager should be sufficiently impatient ( $r$ is large). If the manager is patient, she cares only for the long run in which she ends up with the reputation that she deserves regardless of her earlier attempts to gamble. In this regard, it is worth noticing that condition $r>\frac{\alpha(\alpha-1) a^{2}}{2 \sigma^{2}}$ in $(15)$ is only a sufficient condition for risk shifting. Numerical analysis shows that the manager is tempted to gamble under much milder conditions on $r$ when $a / \sigma^{2}>1 / \alpha$ is satisfied.

To assess whether the risk-shifting friction is likely to be important in practice, we consider a calibration consistent with that of Berk and Green. We set $\alpha=2$, $a=5 \%, \sigma=25 \%$, and $r=5 \%$. Simple calculations then show that the conditions (15) are satisfied. More generally, condition $a / \sigma^{2}>1 / \alpha$ is very likely to be satisfied in practice. It holds whenever the Sharpe ratio of a portfolio strategy is larger than its volatility $(a / \sigma>\sigma)$, which is true for almost all investment strategies. In sum, this suggests that risk shifting matters in this model for parameter values that are empirically plausible. ${ }^{1}$ 


\section{Equilibria with Risk Shifting}

Proposition 4 establishes that under the plausible conditions (15), an equilibrium without gambling does not exist. This raises the following questions: Under these conditions, which gambles emerge in equilibrium? How does gambling in equilibrium affect learning and the distribution of realized returns?

To answer these questions, we consider a reduced-form version of our model. It allows us to abstract from complex signalling issues that would arise in an infinite horizon dynamic model, but nevertheless yields interesting insights into equilibrium gambling strategies. ${ }^{2}$

Specifically, we study the following static model. We assume that the manager makes only one investment decision: She can invest one unit of capital using one of two technologies. She can either use an alpha-generating technology, which produces a gross return

$$
e^{a \theta-\sigma^{2} / 2+\sigma \xi}, \quad \xi \sim N(0,1)
$$

where again $\theta \in\{0 ; 1\}$ is the unknown ability of the manager. Alternatively, she can invest her funds in the risk-shifting technology that enables her to generate a gross return with any arbitrary distribution over $[0,+\infty)$ with unit mean.

Investors and the manager share the common prior beliefs $\pi_{0}$ that the manager is skilled $(\theta=1)$. Upon observing the return $R$ realized by the manager - but without knowing if she gambled or used the alpha-generating technology - investors update

their beliefs about the manager's skills. As before, we denote $\pi_{1}^{R}=\operatorname{Prob}\{\theta=1 \mid R\}$ the investors' posterior belief that $\theta=1$, and refer to it as the manager's reputation.

We assume that the manager invests in order to maximize the sum of her expected current return $R$ and a reduced-form continuation utility that is proportional to her reputation $\pi_{1}^{R}$ :

$$
\max E\left(\gamma R+\pi_{1}^{R}\right), \quad \gamma>0
$$


Assuming a continuation utility that is linear in reputation is commonplace in the literature on career concerns (see, for example, Dasgupta and Prat (2008), Holmstrom and Ricart I Costa (1986), and Scharfstein and Stein (1990)). It is also consistent with what the manager obtains in our dynamic model when the fund size is fixed $(\alpha=1)$.

Lemma 3, similar to Proposition 4, shows that if $a>\sigma^{2}$ then an equilibrium without gambling does not exist.

LEMMA 3: If $a>\sigma^{2}$ and $\pi_{0}$ is sufficiently low, then there exists no equilibrium without gambling.

Proof of Lemma 3: See Appendix.

We now assume that the conditions in Lemma 3 are satisfied, and characterize an equilibrium with gambling. As before, we assume that the manager who is caught gambling is excluded from the market and attains zero utility. Thus, in any equilibrium with gambling, the manager cannot gamble with a probability of one. Notice that if the manager decides to gamble she should choose a distribution for her returns that has no atoms other than at zero. Suppose otherwise that investors believe that a particular return $\hat{R}>0$ occurs with a strictly positive probability. Because the distribution of returns of the efficient technology has no atoms then upon observing $\hat{R}$ investors would conclude that the manager gambles and penalize her with zero payoff. In this case, the manager would be better off by setting $\hat{R}$ to zero in the first place and using the realized surplus to improve the distribution of returns.

The foregoing observations imply that the investment strategy of the manager can be summarized as follows. The manager invests in her alpha-generating technology with probability $(1-q)$ and gambles with probability $q \in(0,1)$. In the latter case, she chooses a gamble that pays off 0 with probability $x \geq 0$ and, conditionally on not yielding 0 , admits an atomless density $\varphi$ over $[0, \infty)$. The following lemma further characterizes this strategy. 
LEMMA 4: The gamble yields 0 with a positive probability $(x>0)$. The density $\varphi$ is single-peaked and has the support $\left[z_{1}, z_{2}\right]$, where $1<z_{1}<z_{2}$.

Proof of Lemma 4: See Appendix.

Lemma 4 shows that equilibria with risk shifting involve trading strategies that consist exactly in taking on hidden-tail risk. With some probability, the manager sells disaster insurance. She uses the insurance premium to generate a noisy excess return if the disaster does not occur. Upon observing such fake excess returns, investors still revise their views about the manager's skills upwards because the manager also uses her alpha-generating technology with some probability.

The proof of Lemma 4 provides an analytical expression for $\varphi$. Here we consider a numerical example. We assume the following parameter values: $\sigma=10 \%, a=2 \sigma^{2}=$ $2 \%, \pi_{0}=40 \%$, and $\gamma=10 \%$.

First, we check that with these parameter values, an equilibrium without gambling does not exist. Suppose, by contradiction, that such an equilibrium does exist. In this case, after observing a return $R$, investors form a posterior belief about the manager's skills as

$$
\pi_{1}^{R}=\frac{\pi_{0} \chi R^{a / \sigma^{2}}}{\pi_{0} \chi R^{a / \sigma^{2}}+1-\pi_{0}}, \quad \chi=e^{-a\left(a-\sigma^{2}\right) / 2 \sigma^{2}} .
$$

Figure 1 Panel (a) depicts $\pi_{1}^{R}$. It is increasing in $R$ and is first convex and then concave.

[Figure 1 About Here]

If the manager does not gamble and invests in the efficient technology, her expected future reputation coincides with the current reputation, and is $\pi_{0}$. The electronic appendix offers a simple procedure to check whether the manager can enhance her expected reputation by resorting to gambling. This procedure consists in finding the straight line that has the smallest value at one among all the straight lines that are above the graph of $\pi_{1}^{R}$. The manager manipulates her reputation if and only if this 
straight line takes a value strictly larger than $\pi_{0}$ at $R=1$. An optimal gamble has its support included in the set where this line coincides with $\pi_{1}^{R}$.

Panel (a) shows that in our example, there is an optimal gamble which delivers either 0 or a gross return of 1.23 with probability of $80 \%$. The expected reputation from such a gamble is $40.6 \%>40 \%$. The gains in expected reputation from gambling come at the cost of the lower expected returns. If the manager gambles, the expected return is only 1 , while it is $\pi_{0} e^{a}+\left(1-\pi_{0}\right)=1.008$ if she uses the alpha-generating technology. Because the cost is less than the reputation gain, an equilibrium without gambling is not sustainable.

Next, we solve for the manager's equilibrium strategy with randomized gambling. Figure 1 Panel (b) shows the equilibrium posterior $\pi_{1}^{R}$. In contrast to that in Panel (a), the posterior now has a linear portion over $[1.02,1.49]$. In equilibrium, the manager gambles with probability $q=5.6 \%$. When she gambles, the manager loses everything with probability $x=8.8 \%$ and obtains an excess return between $2 \%$ and $49 \%$ with a density shown in Figure 1 Panel (c). The range of these returns [1.02, 1.49] corresponds

to the linear part of $\pi_{1}^{R}$ in Figure 1 Panel (b). The unconditional probability that the manager goes bust is $q \times x=0.5 \%$. Thus, the manager trades an excess return with probability $99.5 \%$ with the risk of losing everything.

While binary gambles are optimal deviations in an equilibrium in which investors believe that the manager does not gamble, the equilibrium gambles are not binary because this would be detected by investors. The equilibrium posterior with gambling is flatter than the one without gambling in the region $[1.02,1.49]$, where the returns from gambling are realized. As a result, learning about managerial skills is slower in the equilibrium with gambling.

\section{What Are the Costs of Risk Shifting?}

The results of Sections $B$ and $C$ suggest that there are three types of costs associated with risk shifting induced by career concerns in delegated asset management. First, 
realized returns are riskier in equilibria with gambling. While in our setting investors are risk-neutral, it would be straightforward to extend our model to one in which investors are risk-averse. In this case, additional risk would be costly to them, unless one assumes that gambles are purely idiosyncratic and that investors can diversify them away. From a financial stability perspective (which is beyond the scope of our model), the most worrying aspect of equilibrium risk shifting is that it thickens the left tail of returns, since the manager finances small and frequent positive excess returns with rare but devastating losses. When such incentives prevail within institutions that have legal access to the public safety nets, or that are too big or too systemic too fail, the induced cost for taxpayers of the occurrence of such fat-tailed returns can be very high.

Second, the manager may give up pursuing alpha-generating strategies and invest instead in fairly priced portfolios, which have a risk profile better suited to the manipulation of her reputation. As a result, markets may not be as efficient as they would be if the manager tried to correct any mispricing.

Finally, gambling in equilibrium slows down the discovery of managerial skills because the manager applies them less often. This leads to a less efficient allocation of capital.

\section{Long-Term Contracts and Risk Shifting}

In the previous section we showed that risk shifting reduces the gains from matching "brains and resources" through delegated asset management, and that it generates significant tail risk for financial institutions. It also shows that risk shifting is likely to occur in equilibrium if only one-period contracts are used.

This section introduces the possibility of long-term contracting. We perform two different exercises. First, we consider the risk-taking incentives induced by performance fees with high-water marks, which are commonly used in the hedge fund industry. We 
show that this contract does not eliminate the risk-shifting incentives created by the flow-performance relationship. Second, we solve for optimal contracts that eliminate risk shifting even when conditions (15) are satisfied.

For simplicity, this section restricts the analysis to the case in which the alphagenerating technology is such that $\alpha=1$ in (2). In this case, reputation affects the manager's expected alpha, but not the optimal fund size, which is constant and equal to 1 .

\section{A. Performance Fees with High-Water Marks}

The typical fee structure in the hedge fund industry usually includes a management fee and a performance fee. Management fees are a fixed fraction of the fund's net asset value ranging from $1 \%$ to $4 \%$ per annum, and are meant to cover the fund's operating costs. The performance fee is a fraction of the fund's profits over a given year, ranging from $15 \%$ to $50 \%$. The most widespread combination is a $2 \%$ management fee and a $20 \%$ performance fee, the so-called 2-20 contract. The performance fee typically includes a "high-water mark" (HWM) provision, meaning that the fee applies only to the profits in excess of the previous fund maximum. The goal of this section is to study whether the performance fees are conducive to risk shifting in our model.

Because the terms of the contract are usually fixed, in general the manager does not extract the maximum expected surplus but leaves some surplus to investors. This would not be possible with the one-sided commitment assumed thus far because the manager would enter into a new contract with higher fees if her reputation improved. Such renegotiation by hedge fund managers is not just a theoretical possibility. In practice, managers do adjust their fees in response to their performance. There is also ample anecdotal evidence that fund managers tend to close funds that have underperformed in order to reset the high-water mark. ${ }^{3}$ Nevertheless, in order to provide a meaningful analysis, we only assume in this Section $A$ that both investors and the manager agree on the fees and can commit not to renegotiate them. 
To understand the risk-taking incentives created by the performance fee, we first consider a situation in which there are no new inflows after the manager and investors sign the contract at date 0 . Specifically, we assume that both the manager and investor can commit to the following contract: (i) At date 0, investors make an initial unit investment in the fund; (ii) The manager receives a performance fee $k$ with a highwater mark equal to one and returns the fraction of the fund value in excess of 1 that she does not receive as a performance fee to investors; (iii) There are no new inflows to the fund.

Under the above contract, if the manager uses the alpha-generating technology, the fund size $\left(q_{t}\right)_{t \geq 0}$ evolves as:

$$
\begin{aligned}
d q_{t} & =q_{t}\left[(r+a \theta) d t+\sigma d B_{t}\right]-d H_{t}, \\
H_{t} & =\int_{0}^{t} 1_{\left\{q_{s}=1\right\}} d q_{s} .
\end{aligned}
$$

$H_{t}$ is the fund value in excess of 1 that is redeemed to investors after the performance fee is paid. It is a non-decreasing adapted process that increases only when $q_{t}$ exceeds the optimal fund size equal to 1 . At any date $t$, the continuation utility of the manager is

$$
W_{t}=E_{t}\left[\int_{t}^{\infty} e^{-r s} k d H_{t+s}\right] .
$$

The next proposition computes this utility $W_{t}$ and shows that the manager never finds it worthwhile to gamble in this case.

PROPOSITION 5: Suppose that the manager commits to the contract described in (i), (ii), and (iii) above. Then, she never finds gambling worthwhile and her continuation utility is

$$
\begin{aligned}
W_{t} & =k\left(\pi_{t} \rho^{-1} q_{t}^{\rho}+\left(1-\pi_{t}\right) q_{t}\right), \\
\rho & =\frac{-\left(r+a-\sigma^{2} / 2\right)+\sqrt{\left(r+a-\sigma^{2} / 2\right)^{2}+2 r \sigma^{2}}}{\sigma^{2}}<1 .
\end{aligned}
$$




\section{Proof of Proposition 5: See Appendix.}

Proposition 5 shows that without new inflows, a performance fee with a high-water mark is not sufficiently convex to trigger gambling. A similar result is established by Panageas and Westerfield (2009). Although our results are closely related, our setting differs from that of Panageas and Westerfield along several interesting dimensions.

Panageas and Westerfield also considered a risk-neutral fund manager who is compensated according to (21). The manager cannot secretly gamble but she can take positions in a risk-free asset and in a risky asset that carries an exogenous constant excess return. Panageas and Westerfield found that despite risk neutrality, the manager optimally maintains a finite and constant leverage. One way to understand their result is to notice that the performance fee with high-water mark can be viewed as a continuum of call options with varying maturities. An increase in risk raises the value of the options with the closest maturity but it also increases the possibility that more remote options will become far out-of-the money in case of adverse realizations. The resulting trade-off yields a constant leverage that depends both on the manager's discount rate and on the excess return on the risky asset. When the latter goes to zero, Panageas and Westerfield show that the optimal leverage goes to infinity. This result, however, crucially depends on the assumption that the manager's discount rate is strictly greater than the risk-free rate.

If the manager had a discount rate greater than $r$ in our setting, she would then find it optimal to gamble as her reputation deteriorates. In our model, as the manager's reputation declines, the perceived expected return on the alpha-generating technology goes to zero. Therefore, at some point the manager would choose to forfeit investing in

a technology that provides a small excess return but limited risk in favor of gambling with unrestricted risk-taking possibilities. This does not occur here only because the manager's discount rate is equal to the risk-free rate. In this case, the manager never trades, even in the hope of gaining small expected excess returns in exchange for more risk. 
Proposition 5 may suggest that the HWM contract does not lead to gambling. This conclusion, however, does not apply if fund inflows are considered. The case of $\alpha=1$ is especially illustrative and simple. Provided that the reputation of the manager is high enough to cover her performance fees, investors will always be willing to supply funds until the assets under management reach their optimal size equal to one. This drastically reduces the penalty that accrues from realizing negative returns and therefore, from gambling.

To demonstrate this, suppose that the manager has an option to reopen her fund once to new investors. Let $q^{*}$ be the fund size when she decides to reopen her fund. In this case, the manager raises $1-q^{*}$ units of fresh capital with a high-water mark set to one. We assume that the new investors are the first to withdraw their capital if the total fund size exceeds the optimal fund size of one. ${ }^{4}$

Let $q_{t}^{o}$ and $q_{t}^{n}$ be the respective stakes in the fund of the old and new investors after the fund is reopened and before the stake of the old investors reaches one. Then if the manager uses her alpha-generating technology, $q_{t}^{o}$ and $q_{t}^{n}$ evolve as

$$
\begin{aligned}
d q_{t}^{o} & =q_{t}^{o}\left[(r+a \theta) d t+\sigma d B_{t}\right], \\
d\left(q_{t}^{o}+q_{t}^{n}\right) & =\left(q_{t}^{o}+q_{t}^{n}\right)\left[(r+a \theta) d t+\sigma d B_{t}\right]-d H_{t}, \\
H_{t} & =\int_{0}^{t} 1_{\left\{q_{t}^{o}+q_{t}^{n}=1\right\}} d\left(q_{t}^{o}+q_{t}^{n}\right) .
\end{aligned}
$$

When the stake of the old investors reaches one, they become the sole investors in the fund again, and their capital evolves according to (19) and (20). We denote this moment as $\tau_{1}$. Let $\tau_{q^{*}}$ denote the time when the funds of the original investors reach a level $q^{*}$ and the manager reopens the fund to new inflows. The manager's expected fees from new investors are

$$
W^{n}\left(q^{*}\right)=E_{t}\left[\int_{\tau_{q^{*}}}^{\tau_{1}} e^{-r s} k q_{t}^{n} d H_{t+s}\right],
$$


where $H_{t}$ given by (25). The present value of these fees is

$$
E e^{-r \tau_{q^{*}}} W^{n}\left(q^{*}\right)
$$

While $W^{n}\left(q^{*}\right)$ decreases in $q^{*}$ and reaches maximum at $q^{*}=0, E e^{-r \tau_{q *}}$ increases in $q^{*}$. Thus, in setting $q^{*}$ that solves

$$
\sup _{q^{*}} E e^{-r \tau_{q^{*}}} W^{n}\left(q^{*}\right)
$$

the manager faces a trade-off between the amount of fees she can generate from new investors and how quickly she can receive these fees.

Gambling affects the distribution of $\tau_{q *}$. In particular, if the manager takes on hidden tail risk and realizes a low return, the manager can exercise her option to raise new funds sooner. This greatly reduces the cost of gambling and therefore provides strong incentives to engage in risk-shifting. Of course, the option to raise new funds is only of value to managers whose reputation is good enough that they are still able to attract new flows after a loss. Therefore, incentives to take on hidden-tail risk are highest for managers with a good reputation. However, this result depends on the assumption made in this section that the manager does not renegotiate her contract as her reputation improves. In the next section, we show that when the manager is paid the maximum surplus she can generate, it is conversely a manager with a low reputation who has the highest incentives to gamble. This is because a manager who is perceived to be highly skilled has much to lose if her reputation becomes tarnished. Under the 2/20 contract, however, a good manager does not generally extract the maximum surplus, which makes gambling less costly. We have

PROPOSITION 6: Suppose that the manager commits to the contract described in (i) and (ii). In addition, suppose that the manager has an option to reopen her fund to new investors who supply funds as long as they expect to break even. If $r>\sigma^{2} / 10$ there 
then exists $\bar{\pi}$ such that for all $\pi \geq \bar{\pi}$, the manager with a reputation $\pi$ gambles.

Proof of Proposition 6: See Appendix.

Proposition 6 shows that an option to receive more inflows, even if it occurs only once, is sufficient to create gambling incentives. This suggests that typical performance fees may be problematic in practice given that hedge funds are typically open-ended and do actually have the option to increase their capital.

\section{B. Optimal Contract with Contingent Bonus Deferral}

Under the strong - and in our view unrealistic - assumption that the manager can commit not to renegotiate a contract after her reputation has improved, very simple contracts can eliminate risk shifting. For example, fully insuring the manager by guaranteeing a fixed wage equal to $a \pi_{0}$ per period would eliminate any incentives to gamble. This is not feasible in the more realistic environment with one-sided commitment considered here. The manager can walk away from such an insurance contract as soon as her reputation improves.

When commitment is one-sided, contracts must be structured in such a way that the continuation utility of the manager at any date is at least as large as her outside options given her current reputation. This implies that a contract for a given initial $\pi_{0}$ cannot be determined in isolation. Instead, all contracts for all initial skill levels depend on each other through the channel of managerial outside options. In this section we present contracts that eliminate risk shifting. They exploit the fact that the impact of gambling on investors beliefs vanishes in the long run.

Before constructing these contracts, we illustrate the way they work using the

following simple contract. Suppose that investors and the manager sign the following contract at date 0 . The investors commit to invest one unit in the fund at each date between 0 and $T$. The manager does not receive any payment until date $T$, at which 
she receives a single payment $w_{T}$ such that:

$$
w_{T}=\frac{a \pi_{T}}{r}\left(e^{r T}-1\right)
$$

Suppose, for now only, that after date $T$ the manager no longer works. The date- 0 present value of the payment is

$$
E_{0}\left[e^{-r T} w_{T}\right]=\frac{a \pi_{0}}{r}\left(1-e^{-r T}\right)
$$

which is exactly the present value of the excess returns that investors expect to receive between 0 and $T$ if the manager uses her alpha-generating technology throughout. The contract resembles a deferred bonus with clawback provisions, whereby the manager receives a single terminal payment that is contingent on her entire track record over the period $[0, T]$, as summarized by $\pi_{T}$.

Consider the manager's incentives to gamble at date 0 given such a contract. As in (13), if the manager decides to gamble she chooses a distribution $\mu$ that solves:

$$
\begin{aligned}
\Pi(\pi, T) \equiv & \max _{\mu} \int_{0}^{\infty} E_{0}\left[\frac{\pi_{T} R^{\frac{a}{\sigma^{2}}}}{1-\pi_{T}+\pi_{T} R^{\frac{a}{\sigma^{2}}}} \mid \pi_{0}=\pi\right] d \mu(R) \\
& \text { s.t. } \quad \int_{0}^{\infty} \operatorname{Rd} \mu(R)=1 .
\end{aligned}
$$

The manager will not be tempted to gamble at date 0 if and only if

$$
\Pi\left(\pi_{0}, T\right)=\pi_{0}
$$

The following lemma establishes important properties of $\Pi(\pi, T)$.

LEMMA 5: For all $\pi \in(0,1)$, the function $\Pi(\pi, T)$ decreases with respect to $T$, and is equal to $\pi$ for all $T$ sufficiently large. Furthermore, there exists $\underline{\pi}$ such that for all $\pi \geq \underline{\pi}, \Pi(\pi, 0)=\pi$. 
Proof of Lemma 5: See Appendix.

Because $\Pi(\pi, T)$ decreases with respect to $T$, date-0 gambling becomes less appealing as the payment date becomes more remote. This formalizes the insight that the impact of gambling on investors' beliefs diminishes over time. Lemma 5 implies in particular that we can define for all $\pi$

$$
\tau(\pi) \equiv \inf \{t \geq 0: \Pi(\pi, t)=\pi\}
$$

If $T \geq \tau\left(\pi_{0}\right)$ then the manager does not gamble at date 0 . As the reputation of the manager improves, incentives to gamble decrease. Lemma 5 shows that a manager with a sufficiently high reputation does not gamble even if the payment is made at date- 0 .

In light of the foregoing, we are now ready to define an optimal contract. There are two matters that the fixed-date single-payment contract outlined above does not address. First, while setting the payment date at $\tau\left(\pi_{0}\right)$ deters date- 0 gambling, there is no guarantee that the manager will not be tempted to gamble as the payment date approaches. Second, it is necessary to check that if the manager enters into a new singlepayment contract after the current one expires, this does not affect her incentives to gamble within the current contract.

We construct the optimal contract as follows. At date 0 , the investors commit to pay $w_{T_{0}}=\frac{a \pi_{T_{0}}}{r}\left(e^{r T_{0}}-1\right)$ at a random date $T_{0}$ which is defined as follows. Initially, $T_{0}$ is equal to $\tau\left(\pi_{0}\right)$. Then for all $n \geq 1$, if $T_{(n-1) \Delta t}=1$, then the payment is made and the contract ends. Otherwise, the date is revised as $T_{n \Delta t}=\max \left\{T_{(n-1) \Delta t}-1 ; \tau\left(\pi_{n \Delta t}\right)\right\}$. After the payment of this current contract is made, a new similar contract is initiated at date $T$ with initial reputation $\pi_{T}$. We have:

PROPOSITION 7: If the manager is compensated according to a sequence of singlepayment contracts such that a contract that starts at date $t$ promises the payment $\frac{a \pi_{T}}{r}\left(e^{r T}-1\right)$ at a random date $T$ defined as above, she never gambles and extracts the maximum expected surplus. 
Proof of Proposition 7: Investors can commit to this contract because by construction, investors break even ex-ante. We therefore only need to prove two remaining results: first that the manager's continuation utility is higher than her outside option at any date, and second that the manager never finds it optimal to gamble.

In respect of the first point, we define the random payment date of the current contract as $T$. At date $t$, the manager expects to receive:

$$
E_{t}\left[e^{-r(T-t)}\left(w_{T}+\frac{a \pi_{T}}{r}\right)\right]=\frac{e^{r t} a \pi_{t}}{r} \geq \frac{a \pi_{t}}{r} .
$$

In respect of the second point, we observe that by construction, the random payment date $T$ is such that the manager has no incentive to gamble in order to increase the expected payment from the current contract. It may still be the case that the manager finds it worthwhile to gamble to increase her expected payoff on the subsequent contracts that follow the terminal payment of the current one. Notice that from Lemma 5, the case that is the most conducive to gambling is if all the payments from subsequent contracts were made once and for all at date $T$. Even in this case, the expected payment is $\frac{a \pi_{T}}{r}$, which is proportional to $w_{T}=\frac{a \pi_{T}}{r}\left(e^{r T}-1\right)$, and thus not conducive to gambling.

Q.E.D.

Notice that this contract can be implemented in practice with share grants to the manager instead of cash bonuses provided that i) the manager is sufficiently senior or important within the firm that her decisions actually affect the share price, ii) the vesting schedule is stochastic, depending on the manager's entire track record as described above.

It is interesting to compare this contract with the one that emerges from models of dynamic moral hazard such as, for example, DeMarzo and Sannikov (2006) or He (2009). In these models, an agent may secretly divert cash flows instead of reporting her entire output to the principal. The optimal contract in this case consists, as in 
ours, in adjusting the present value of the future payments promised to the agent as her track record evolves.

However, the timing conditions of the future payments are rather different in those contracts compared with ours. In our contract it is crucial that the investors can commit to not accelerating the timing of payments upon observing a stellar performance. In moral-hazard models, a stellar track record results in accelerated payments being made to the agent.

This difference arises because the contracts are driven by different economic considerations. In our model, a stream of fixed promised payments would completely eliminate any risk-shifting incentives. However, such fixed payments are not viable because investors are forced to adjust the manager's continuation value as her reputation improves. If not, a good track record would lead the manager to repudiate her current labor contract and sign a new one that reflects her new improved reputation. The necessity of adjusting the continuation value creates risk-shifting incentives that can be addressed by the investors' commitment to deferring payments.

In contrast, in moral-hazard models, an adjustment of continuation values is meant to provide the agent with incentives to report the highest possible output instead of diverting some of it. Thus, the sooner the agent reports high income, the better.

\section{Current Financial Reforms and Contingent Bonus Deferral Con- tract}

There is a widely shared view that inappropriate compensation schemes within the financial services industry were one of the ingredients that led to the financial crisis that erupted in 2008. According to "Principles for Sound Compensation Practices," published by the Financial Stability Forum (FSF) and summarizing the outcome of multiple surveys of financial institutions "over 80 percent of market participants believe that compensation practices played a role in promoting the accumulation of risks that 
led to the current crisis."

In the face of these compensation issues, public authorities around the world have issued guidelines for compensation reforms. These guidelines prominently feature the deferral of bonuses and the introduction of clawback mechanisms. For example, in the U.S., the Guidance on Sound Incentive Compensation Policies, jointly issued by several authorities, ${ }^{5}$ lists four methods of making compensation more sensitive to risk, including "deferral of payment" with explicit mention of clawbacks, "longer performance periods", and a "reduced sensitivity to short-term performance". The Financial Stability Forum points to similar tools, and writes: "One way to align time horizons is to place a portion, and in some cases up to the entirety, of any given year's bonus grant, both cash and equity, into the equivalent of an escrow account. All or part of the grant is reversed if the firm as a whole performs poorly, or if the exposures the employee caused the firm to assume in the year for which the bonus was granted perform poorly (a clawback)."

The industry is gradually beginning to follow this guidance. The International Institute of Finance, surveying the practices of 37 financial institutions representing more than half of global banking activity, concludes ${ }^{6}$ : "The industry has begun to take steps to strengthen the link between delivery of deferred compensation and the continued performance of the individual. Over $40 \%$ of the firms surveyed include performancebased criteria in their deferred compensation schemes, although in a majority of cases this takes simply the form of a penalty for gross misconduct or large-scale unexpected losses. A number of firms have developed more sophisticated approaches that incorporate a final payout multiplier that adjusts compensation up or down based on current year or historical performance."

Interestingly, the stochastic payment dates that we introduce in this section strongly resemble this introduction of bonus deferral and clawbacks. While our optimal contract follows the spirit of these reforms, we believe that it also introduces some important caveats. Our model suggests that setting the bonus payment date once and for all is in 
general not optimal, even if the terminal bonus is adjusted for subsequent performance. Additionally, it is important to commit to adjusting the payment date as events unfold. For example, our analysis shows that a three-year deferral might induce excessive risk taking at the end of year 2 if the manager has not performed well and the bonus is due in one year.

\section{Conclusion}

Financial innovation has come under severe criticism after the crisis that erupted in 2007 . Several papers have documented that a number of structured products appeared mostly to be aimed at exploiting investors' weaknesses or ignorance. ${ }^{7}$ In this paper, we suggest that by enlarging the set of financial instruments available to fund managers, financial innovation may exacerbate agency costs even when investors are sophisticated, provided that investors cannot perfectly monitor the positions of the fund managers.

We introduce a novel framework to study this agency problem between managers and investors. In this framework, managers' compensation depends on investors' perception of their ability to generate excess returns above a fair compensation for risk (alpha). The managers can temporarily distort the perception of these alpha-generating skills by trading a rich menu of financial instruments.

The model delivers two main implications. First, in the absence of careful contracting, this friction may lead managers to take on hidden-tail risk in order to distort their perceived skills temporarily. Second, while current compensation reforms based on the deferral and clawback of bonuses appear to be an appropriate tool for addressing this issue, in their current form these reforms miss the point that the payment dates must be dynamically adjusted as the cumulative performance of the manager evolves.

In general, there are ways of dealing with risk shifting in delegated asset management other than through the design of compensation. Possible solutions include increased transparency, or restrictions on the set of instruments that managers can 
trade. In this paper we focus on solving the risk-shifting problem using compensation design alone. This makes the problem more challenging. Future research could combine optimal contracting with these additional means of addressing the risk-shifting problem. On the other hand, we assume that positions are always valued at a fair market price. Instruments that are more difficult to value such as illiquid securities or exotic derivative contracts are likely to provide fund managers with additional riskshifting incentives if trading losses can be concealed for some time. Future research could further explore these channels in an attempt to engage in risk transformation. 


\section{Notes}

${ }^{1}$ Our results are also broadly supported by empirical findings of Brown, Harlow, and Starks (1996) and Chevalier and Ellison (1997), who show that mutual fund managers tend to take on more risk following their disappointing performance.

${ }^{2}$ In an equilibrium with gambling, unlike in an equilibrium without gambling, the manager and investors possess different information about the manager's ability. While the manager knows whether she gambled or not, investors can only imperfectly infer this from the observed returns. This creates room for signaling, whereby a truly skilled manager would like to distinguish herself credibly from a lucky gambler. Solving for such equilibria with asymmetric information is a very difficult problem and is beyond the scope of this paper.

${ }^{3}$ See, for example, G. Zuckerman, "Andor Haunted by a Bad Bet," July 15th, 2004, Wall Street Journal; A. Stone, "Hedge Funds: Fees Down? Close Shop," Bloomberg Business Week, August 7th, 2005; or "Hedge-Fund Closures: Quitting While They're Behind," The Economist, February 18th, 2012.

${ }^{4}$ This assumption makes gambling less likely because it decreases the manager's expected fees from opening the fund to new investors, and thus makes gambling more costly.

${ }^{5}$ Office of the Comptroller of the Currency, Federal Reserve, Federal Deposit Insurance Corporation, Treasury, and Office of Thrift Supervision.

${ }^{6}$ In "Compensation in Financial Services, Industry Progress and the Agenda for Change," March 2009. The International Institute of Finance is the leading global association of financial institutions. 
${ }^{7}$ For example, Coval, Jurek, and Stafford (2009a) and Coval, Jurek, and Stafford (2009b) contended that senior CDO tranches aimed at exploiting the misperception of correlation risk by rating agencies, and that of systematic exposure by investors. Henderson and Pearson (2011) considered a class of structured equity products offered at prices that are hard to reconcile with purchasers' rationality. 


\section{References}

Acharya, V., Cooley, T., Richardson M., and Walter, I. 2010. "Manufacturing Tail Risk: A Perspective on the Financial Crisis of 2007-09," Foundations and Trends in Finance, Vol. 4: No. 4, 247-325.

Acharya, V., Pagano, M., and Volpin, P., 2012. "Seeking Alpha: Excess Risk Taking and Competition for Managerial Talent," working paper

Basak, S., Pavlova, A., and Shapiro, A., 2007. "Optimal Asset Allocation and Risk Shifting in Money Management”, Review of Financial Studies 20(5), 1583-1621.

Berk, J.B. and Green, R.C., 2004. "Mutual Fund Flows and Performance in Rational Markets," Journal of Political Economy, 112(6), 1269-1295.

Berk, J.B. and Stanton R., 2007. "Managerial Ability, Compensation, and the ClosedEnd Fund Discount," Journal of Finance 62(2), 529-556.

Carpenter, J.N., 2000. "Does Option Compensation Increase Managerial Risk Appetite?," Journal of Finance, 55(5), 2311-2331.

Coval, J., Jurek, J., and Stafford, E., 2009. "The Economics of Structured Finance," Journal of Economic Perspectives 23(1),, 3-25.

Coval, J., Jurek, J., and Stafford, E., 2009. "Economic Catastrophe Bonds," The American Economic Review 99(3), 2009, 628-666.

Cox, D. R., Miller, H. D., 1965. "The Theory of Stochastic Processes," London: Chapman and Hall.

Dangl T., Wu, Y. and Zechner, J., 2008. "Market Discipline and Internal Governance in the Mutual Fund Industry", The Review of Financial Studies, 21, 2307-2343. 
Dasgupta A. and Prat, A., 2008. "Information aggregation in financial markets with career concerns", Journal of Economic Theory, 143, 83-113.

DeMarzo, P., and Sannikov, Y., 2006. "Optimal Security Design and Dynamic Capital Structure in a Continuous-Time Agency Model," The Journal of Finance, 61, 26812724 .

Driver, K.B., 2003. "Analysis Tools With Applications," Lecture Notes.

Goetzmann W., Ingersoll J., Spiegel M., and Welch I., 2007. "Portfolio Performance Manipulation and Manipulation-proof Performance Measures," Review of Financial Studies, 20(5), 1503-1546.

He, Z., 2009. "Optimal Executive Compensation when Firm Size Follows Geometric Brownian Motion," The Review of Financial Studies 22, 859-892.

Henderson, B., and Pearson, N. , 2011. "The Dark Side of Financial Innovation: A Case Study of the Pricing of a Retail Financial Product," Journal of Financial Economics $100,227-247$.

Holmstrom, B. and Ricart I Costa, J., 1986. "Managerial Incentives and Capital Management," The Quarterly Journal of Economics, 101 (4), 835-860.

Jensen, M., and Meckling, W., 1976. "Theory of the Firm: Managerial Behavior, Agency Costs and Ownership Structure," Journal of Financial Economics, 3(4), 305-360.

Jiang, H., and Kelly, B., 2012. "Tail Risk and Hedge Fund Returns," Chicago Booth Paper No. 12-44.

Liptser, R. Sh. and Shiryaev, A. N., 1978. "Statistics of random processes," Springer.

Malliaris, S., and Yan, H., 2012. "Reputation Concerns and Slow-Moving Capital," working paper. 
Panageas, S., and Westerfield, M., 2009. "High-Water Marks: High Risk Appetites? Convex Compensation, Long Horizons, and Portfolio Choice," The Journal of Finance, 64, 1-36.

Rajan, R., 2008. "Bankers' Pay Is Deeply Flawed,” Financial Times, January 9.

Ross, S., 2004. "Compensation, Incentives, and the Duality of Risk Aversion and Riskiness," Journal of Finance, 59(1), 207-225.

Scharfstein, D. and Stein, J., 1990. "Herd Behavior and Investment," The American Economic Review, 80(3), 465-79. 


\section{Appendix. Proofs}

Proof of Lemma 1: As $\Delta t \rightarrow 0$, the optimal fund size and maximal expected surplus solve

$$
\begin{aligned}
& \lim _{\Delta t \rightarrow 0} q(\pi)=\arg \max _{q} q\left(\pi a-\beta q^{\frac{1}{\alpha-1}}\right), \\
& \lim _{\Delta t \rightarrow 0} v(\pi)=\max _{q} q\left(\pi a-\beta q^{\frac{1}{\alpha-1}}\right) .
\end{aligned}
$$

Direct computations show that

$$
\begin{aligned}
& \lim _{\Delta t \rightarrow 0} q(\pi)=\left(\frac{\alpha-1}{\alpha \beta} a \pi\right)^{\alpha-1}, \\
& \lim _{\Delta t \rightarrow 0} v(\pi)=\beta^{1-\alpha} \frac{(\alpha-1)^{\alpha-1}}{\alpha^{\alpha}}(a \pi)^{\alpha} .
\end{aligned}
$$

Q.E.D.

Proof of Proposition 1: We first show point-wise convergence. That is, we establish (6) for a fixed $\pi_{0}=\pi$. By Bayes' theorem, $\pi_{n \Delta t}$, the perceived skills at date $n \Delta t$, satisfy

$$
\pi_{n \Delta t}=\frac{\pi_{0} \varphi_{n \Delta t}}{1-\pi_{0}+\pi_{0} \varphi_{n \Delta t}}
$$

where

$$
\varphi_{n \Delta t}=\exp \left\{\frac{a}{\sigma^{2}}\left(a\left(\theta-\frac{1}{2}\right) n \Delta t+\sigma B_{n \Delta t}\right)\right\}
$$

is the likelihood ratio process. Let us introduce the continuous-time process $\left(\pi_{t}\right)_{t \geq 0}$ that obeys

$$
d \pi_{t}=\frac{a}{\sigma} \pi_{t}\left(1-\pi_{t}\right) d \bar{B}_{t}, \quad \pi_{0}=\pi
$$

where $\bar{B}_{t}=\frac{1}{\sigma}\left(\theta a t+\sigma B_{t}-a \int_{0}^{t} \pi_{s} d s\right)$. Then $\left(\bar{B}_{t}\right)_{t \geq 0}$ is a standard Wiener process under the agents' filtration (see Liptser and Shiryaev (1978)). Further, as $\Delta t \rightarrow 0$ and $n \Delta t \rightarrow t, \pi_{n \Delta t} \rightarrow \pi_{t}$ a.s. (see Liptser and Shiryaev (1978)). Hence, $V(\pi)$ can be 
written as

$$
\begin{aligned}
V(\pi) & =E_{0} \int_{0}^{\infty} e^{-r t} v\left(\pi_{t}\right) d t \\
\text { s.to } d \pi_{t} & =\frac{a}{\sigma} \pi_{t}\left(1-\pi_{t}\right) d \bar{B}_{t}, \quad \pi_{0}=\pi .
\end{aligned}
$$

By the Feynman-Kac formula, the function $V$ solves the following linear second-order differential equation:

$$
\frac{a^{2}}{2 \sigma^{2}} \pi^{2}(1-\pi)^{2} V^{\prime \prime}(\pi)-r V(\pi)+v(\pi)=0
$$

From (A3) it follows that

$$
V(0)=v(0) / r, \quad V(1)=v(1) / r .
$$

The corresponding homogeneous equation

$$
\frac{a^{2}}{2 \sigma^{2}} \pi^{2}(1-\pi)^{2} V^{\prime \prime}(\pi)-r V(\pi)=0
$$

has two regular singular points at 0 and 1 . All solutions of the homogeneous equation are linear combinations of the two independent solutions

$$
\begin{aligned}
g(\pi) & =(1-\pi)^{\frac{1}{2}+\frac{1}{2} \psi} \pi^{\frac{1}{2}-\frac{1}{2} \psi}, \quad \psi=\sqrt{1+8 r \sigma^{2} / a^{2}} \\
h(\pi) & =g(1-\pi) .
\end{aligned}
$$

From here, formulas (6) and (7) are standard results in the theory of inhomogeneous differential equations. The function $G$ is the Dirichlet-Green function for the differential operator associated with the homogeneous differential equation (see, for example, Driver (2003)).

We now show that $V(\pi, \Delta t)$ converges to $V(\pi)$ uniformly in $\pi$ as $\Delta t \rightarrow 0$. We have 


$$
V(\pi)-V(\pi, \Delta t)=E\left[\sum_{n=0}^{\infty} \int_{n \Delta t}^{(n+1) \Delta t}\left(e^{-r t} v\left(\pi_{t}\right)-e^{-r n \Delta t} v\left(\pi_{n \Delta t}\right)\right) d t \mid \pi_{0}=\pi\right]
$$

Thus, it is enough to show that $\forall \varepsilon>0, \exists \bar{\Delta} t$ such that $\forall \Delta t<\bar{\Delta} t$ and $\forall \pi \in[0,1]$

$$
\sup _{s \leq \Delta t} \sup _{\pi \in[0,1]}\left|E\left(v\left(\pi_{s}\right)-v(\pi)\right)\right|<\varepsilon
$$

By change of variables (A7) can be written as

$$
\sup _{s \leq \Delta t} \sup _{\pi \in[0,1]}\left|E\left(\widehat{v}\left(\pi, B_{s}, s\right)-\widehat{v}(\pi, 0,0)\right)\right|<\varepsilon
$$

where

$$
\widehat{v}(\pi, x, t)=v\left(\frac{\pi \exp \left\{\frac{a}{\sigma^{2}}\left(a\left(\theta-\frac{1}{2}\right) t+\sigma x\right)\right\}}{1-\pi+\pi \exp \left\{\frac{a}{\sigma^{2}}\left(a\left(\theta-\frac{1}{2}\right) t+\sigma x\right)\right\}}\right) .
$$

Because $v$ is uniformly continuous over $[0,1]$ it is enough to show that $\forall \pi \in[0,1]$ and $\forall \varepsilon>0, \exists \bar{\Delta} t$ such that $\forall \Delta t<\bar{\Delta} t$

$$
\sup _{s \leq \Delta t}\left|E\left(\widehat{v}\left(\pi, B_{s}, s\right)-\widehat{v}(\pi, 0,0)\right)\right|<\varepsilon
$$

This follows from the weak convergence of the measures induced by $B_{s}$ to the measure concentrated at 0 as $s \rightarrow 0$.

Q.E.D.

Proof of Proposition 2: Suppose that investors believe that the manager invests in her private technology. In this case, if the manager does invest in her private technology then $\pi_{t}$ evolves according to (A3). If, on the other hand, she invests in the risk-free asset, $\pi_{t}$ evolves as

$$
d \pi=\frac{a}{\sigma} \pi(1-\pi)\left(r^{\prime}-r+\frac{\sigma^{2}}{2}-\left(\pi a-c\left(q_{t}\right)\right)\right) d t
$$


Suppose that at time $t$ the manager allocates $x_{t}$ percentage of her funds to her alphagenerating technology and invests the rest in the risk-free asset. Then her continuation utility is

$$
\begin{aligned}
V(\pi, x) & =E_{0} \int_{0}^{\infty} e^{-r t} v\left(\pi_{t}\right) d t \\
\text { s.to } d \pi_{t} & =\frac{a}{\sigma} \pi_{t}\left(1-\pi_{t}\right)\left(\left(1-x_{t}\right)\left(r^{\prime}-r+\frac{\sigma^{2}}{2}-\left(\pi a-c\left(q_{t}\right)\right)\right)+x_{t} d \bar{B}_{t}\right), \pi_{0}=\pi .
\end{aligned}
$$

The optimal investment policy $x_{t}$ that maximizes $(A 10)$ satisfies the HJB equation:

$$
\sup _{x \in[0,1]} x^{2} \psi_{2}^{2} V^{\prime \prime}+(1-x) \psi_{1} V^{\prime}-r V+v=0
$$

where

$$
\begin{aligned}
\psi_{1} & =\frac{a}{\sigma} \pi_{t}\left(1-\pi_{t}\right)\left(r^{\prime}-r+\frac{\sigma^{2}}{2}-\left(\pi a-c\left(q_{t}\right)\right)\right)<0 \\
\psi_{2} & =\frac{a}{\sigma} \pi_{t}\left(1-\pi_{t}\right)>0
\end{aligned}
$$

If $x_{t} \equiv 1$ then $\pi_{t}$ is a martingale and by Jensen's inequality $\left(v(\pi)=\pi^{\alpha}\right)$

$$
E v\left(\pi_{t}\right) \geq v\left(\pi_{0}\right)
$$

Therefore, at the optimal investment policy $x_{t}, r V(\pi, x) \geq v(\pi)$. Thus (A11) implies that the optimal policy is indeed $x_{t} \equiv 1$.

Q.E.D.

Proof of Proposition 3: Suppose that the manager gambles and realizes a return $R$ over $[0, \Delta t]$, and from then on invests in her alpha-generating technology. Let $\left(\pi_{n \Delta t}^{R}\right)_{n \in \mathbb{N}}$ denote the process under the manager's filtration of her skills as perceived by investors who believe instead that she has invested in her storage technology at date 0 . These 
investors believe that

$$
R=e^{\left(r+\theta a-c\left(q_{0}\right)-\frac{\sigma^{2}}{2}\right) \Delta t+\sigma B_{\Delta t}} .
$$

From (A1) and (A2) it follows that

$$
\begin{aligned}
& \pi_{\Delta t}^{R}=\frac{\left.\pi_{0} R^{\frac{a}{\sigma^{2}}} e^{\frac{a}{\sigma^{2}}} \frac{\sigma^{2}}{2}+c\left(q_{0}\right)-r-\frac{a}{2}\right) \Delta t}{1-\pi_{0}+\pi_{0} R^{\frac{a}{\sigma^{2}}} e^{\frac{a}{\sigma^{2}}\left(\frac{\sigma^{2}}{2}+c\left(q_{0}\right)-r-\frac{a}{2}\right) \Delta t}}, \\
& \forall n \geq 0, \quad \pi_{(n+1) \Delta t}^{R}=\frac{\pi_{\Delta t}^{R} \frac{\varphi(n+1) \Delta t}{\varphi \Delta t}}{1-\pi_{\Delta t}^{R}+\pi_{\Delta t}^{R} \frac{\varphi(n+1) \Delta t}{\varphi \Delta t}} .
\end{aligned}
$$

As $\Delta t \rightarrow 0$

$$
\lim _{\Delta t \rightarrow 0} \pi_{\Delta t}^{R}=\frac{\pi_{0} R^{\frac{a}{\sigma^{2}}}}{1-\pi_{0}+\pi_{0} R^{\frac{a}{\sigma^{2}}}},
$$

and

$$
\lim _{\Delta t \rightarrow 0} \frac{\varphi_{(n+1) \Delta t}}{\varphi_{\Delta t}}=\frac{\pi_{t}}{1-\pi_{t}} \frac{1-\pi_{0}}{\pi_{0}} .
$$

Therefore,

$$
\lim _{\Delta t \rightarrow 0, n \Delta t \rightarrow t} \pi_{n \Delta t}^{R}=\frac{\pi_{t} R^{\frac{a}{\sigma^{2}}}}{1-\pi_{t}+\pi_{t} R^{\frac{a}{\sigma^{2}}}} .
$$

Q.E.D.

Proof of Proposition 4: Part 1. We first show that if $\sigma^{2}>\alpha a$ then the manager does not engage in risk shifting. Proposition 2 demonstrates that the manager will not invest in the alternative technology at the risk-free rate, provided that $r^{\prime}<r-\sigma^{2} / 2$. We now show that she will not invest in any risky gamble as well.

Suppose the manager believes that she is skilled with probability $\pi$. At the same time, suppose that investors believe that the manager is skilled with probability $\pi^{\prime}$ and that the manager never engages in risk shifting. We show that the manager has no incentives to deviate by taking a one-shot risky gamble in this case.

Suppose the manager takes a gamble and realizes return $R$. Let $W\left(\pi, \pi^{\prime}, R, \Delta t\right)$ be the expected utility of the manager conditional on realization a first-period return $R$. Similar to the proof of Proposition 3 one can show that investors' perception of the 
manager's skills $\pi_{t}^{R}$ is

$$
\pi_{t}^{R}=\frac{\pi_{t} R^{\frac{a}{\sigma^{2}}}}{\left(1-\pi_{t}\right) \frac{\left(1-\pi^{\prime}\right) \pi}{(1-\pi) \pi^{\prime}}+\pi_{t} R^{\frac{a}{\sigma^{2}}}} .
$$

Proposition 1 implies that

$$
\lim _{\Delta t \rightarrow 0} W\left(\pi, \pi^{\prime}, R, \Delta t\right)=\int_{0}^{1} G(\pi, x) v\left(\frac{x R^{\frac{a}{\sigma^{2}}}}{(1-x) \frac{\left(1-\pi^{\prime}\right) \pi}{(1-\pi) \pi^{\prime}}+x R^{\frac{a}{\sigma^{2}}}}\right) d x
$$

Differentiating twice w.r.t. $R$ shows that this function is concave in $R$ when $\sigma^{2} \geq \alpha a$. Hence the manager has no incentives to take a one-shot risky gamble in this case. Because this holds for arbitrary heterogeneous priors $\pi, \pi^{\prime}$, this implies that multiperiod deviations cannot be desirable by backward induction.

Part 2. We now show that if $\sigma^{2}<\alpha a, r \sigma^{2}>\frac{a^{2}}{2} \alpha(\alpha-1)$ then for $\pi_{0}=\pi$ small enough, there exists a one-period gamble which makes the manager better off. Let $R=(1-\rho)^{-\sigma^{2} / a}, \rho \in[0,1)$. Consider the following gamble:

$$
\left\{\begin{array}{lll}
R & \text { Prob. } & 1 / R \\
0 & \text { Prob. } & 1-1 / R
\end{array}\right.
$$

From (A15) the expected net gain from the above one-period gamble over perpetual investment in the efficient storage technology is

$$
\int_{0}^{1} G(\pi, x) x^{\alpha} u(x, \rho) d x
$$

where

$$
u(x, \rho)=\frac{(1-\rho)^{\sigma^{2} / a}}{(1-\rho(1-x))^{\alpha}}-1
$$

Since $\sigma^{2}<\alpha a$, there exists $\bar{x}$ and some $\hat{\rho} \in(0,1)$ such that for all $x \in[0, \bar{x}]$, $\frac{(1-\widehat{\rho})^{\sigma^{2} / a}}{(1-\widehat{\rho}(1-x))^{\alpha}}>1+\varepsilon$ for some $\varepsilon>0$ and therefore, $u(x, \widehat{\rho})>\varepsilon>0$. Thus for $\pi$ small enough

$$
\int_{0}^{1} G(\pi, x) x^{\alpha} u(x, \widehat{\rho}) d x>\int_{\pi}^{1} G(\pi, x) x^{\alpha} u(x, \widehat{\rho}) d x
$$


Using (7) we have

$$
\int_{\pi}^{1} G(\pi, x) x^{\alpha} u(x, \widehat{\rho}) d x=\frac{2 \sigma^{2}}{\psi a^{2}} g(\pi) \int_{\pi}^{1} x^{\alpha-\frac{3}{2}-\frac{1}{2} \psi}(1-x)^{-\frac{3}{2}+\frac{1}{2} \psi} u(x, \widehat{\rho}) d x
$$

$r \sigma^{2}>\frac{a^{2}}{2} \alpha(\alpha-1)$ implies that $\psi>2 \alpha-1$. Therefore, the integral

$$
\int_{\pi}^{1} x^{\alpha-\frac{3}{2}-\frac{1}{2} \psi}(1-x)^{-\frac{3}{2}+\frac{1}{2} \psi} u(x, \widehat{\rho}) d x
$$

diverges as $\pi \rightarrow 0$. In this case, its sign is determined by the sign of $u(\cdot, \widehat{\rho})$ in the neighborhood of 0 , which is positive. Thus, the net gain from the gamble is positive.

Q.E.D.

Proof of Lemma 3: Suppose that investors believe that the manager does not gamble. Then their posterior probability about her ability upon observing $R$ is

$$
\pi_{1}(R)=\frac{\pi_{0} \chi R^{a / \sigma^{2}}}{\pi_{0} \chi R^{a / \sigma^{2}}+1-\pi_{0}}, \quad \chi=e^{-a\left(a-\sigma^{2}\right) / 2 \sigma^{2}}
$$

If the manager does not gamble, she expects to get

$$
\gamma\left(\pi_{0} e^{a}+1-\pi_{0}\right)+\pi_{0}
$$

Consider $\pi_{0}$ such that

$$
4 \pi_{0}\left(1-\pi_{0}\right)<\chi\left(1+\gamma\left(e^{a}-1\right)\right)^{-2}
$$

Suppose that the manager deviates and enters into a gamble that generates gross return $\sqrt{\frac{1-\pi_{0}}{\pi_{0} \chi}}$ with probability $1 / \sqrt{\frac{1-\pi_{0}}{\pi_{0} \chi}}$ and 0 otherwise. Direct computations show that in this case, the manager expects to get

$$
\gamma+\frac{1}{2} \sqrt{\frac{\pi_{0} \chi}{1-\pi_{0}}}
$$


Comparing (A21) with (A19) one can see that the manager obtains a higher utility if she gambles, provided that (A20) holds.

Q.E.D.

Proof of Lemma 4: We solve for an equilibrium in which the manager invests in her private technology with probability $1-q$ and gambles otherwise. If the manager gambles, she receives 0 with probability $x$ and a return between $z$ and $z+d z$ with probability $(1-x) \varphi(z) d z$. For the ease of computations, we write $\varphi(z)$ as

$$
\varphi(z)=\frac{1}{z \sqrt{2 \pi \sigma^{2}}} e^{-\ln ^{2}(z) / 2 \sigma^{2}} g(z)
$$

where $g(z) \geq 0$. In such an equilibrium, upon observing a realized return $z$, investors' posterior is ${ }^{8}$

$$
\pi_{1}(z)=\pi_{0} \frac{(1-q) \chi z^{a / \sigma^{2}}+q(1-x) g(z)}{(1-q)\left(\pi_{0} \chi z^{a / \sigma^{2}}+1-\pi_{0}\right)+q(1-x) g(z)}, \quad \chi=e^{-a\left(a-\sigma^{2}\right) / 2 \sigma^{2}} .
$$

Let $E_{\mu}\left(E_{0}\right)$ be the expectation operator if the manager gambles (invests in her alpha-generating technology). When the manager gambles she takes the investors' posterior $\pi_{1}$ in (A23) as given and chooses a gamble that solves

$$
\begin{aligned}
& \sup E_{\mu} \pi_{1}(z) \\
& \text { s.to } E_{\mu} z=1 .
\end{aligned}
$$

We show in the electronic appendix that the solution to this problem coincides with the solution to its dual problem:

$$
\begin{aligned}
& \inf _{(A, B) \in \mathbb{R}^{2}} A+B \\
& \text { s.to } \forall z \geq 0, A+B z \geq \pi_{1}(z),
\end{aligned}
$$


where $A$ and $B$ are some real numbers.

The dual problem admits a simple and practical interpretation: it minimizes the value at one of a straight line that is above the graph of $\pi_{1}(z)$. The optimal gamble then has its support included in the set where this line coincides with $\pi_{1}(z)$. We also show in the electronic appendix that this solution can be realized with a binary gamble. In this particular case, a binary gamble cannot be the equilibrium solution, however, because it would be detected. Thus it must be that $\pi_{1}(z)$ has linear portions that coincide with the minimal straight line $A+B z$.

Given that $\pi_{1}(z)$ is first convex then concave, it is easy to see that there must be two real numbers $z_{1}$ and $z_{2}$ such that $\pi_{1}(z)=B z$ for $z \in 0 \cup\left[z_{1}, z_{2}\right]$ and $\pi_{1}(z)<B z$ otherwise. Figure 1 Panel (b) illustrates this for a particular numerical example. The interval $\left[z_{1}, z_{2}\right]$ is the support of the gamble when it does not yield 0 .

We are now ready to solve for the manager's gambling strategy. We first determine B. Direct computations show

$$
q E_{\mu} \pi_{1}(z)+(1-q) E_{0} \pi_{1}(z)=\pi_{0}(1-q x)
$$

Because the manager randomizes between gambling and investing in her alpha-generating technology, it must be that she is indifferent between gambling or investing efficiently:

$$
E_{\mu}\left(\gamma z+\pi_{1}(z)\right)=E_{0}\left(\gamma z+\pi_{1}(z)\right)
$$

or

$$
E_{\mu} \pi_{1}(z)=E_{0} \pi_{1}(z)+\gamma \pi_{0}\left(e^{a}-1\right)
$$

(A25) and (A26) together imply that

$$
B=E_{\mu} \pi_{1}(z)=(1-q x) \pi_{0}+\gamma(1-q) \pi_{0}\left(e^{a}-1\right) .
$$


Since $\pi_{1}(z)=B z$ for $z \in 0 \cup\left[z_{1}, z_{2}\right]$, we can solve for $g(z)$ from (A23):

$$
g(z)= \begin{cases}\frac{(1-q) \pi_{0} \chi z^{a / \sigma^{2}}\left(B z+B\left(1-\pi_{0}\right)\left(\pi_{0} \chi\right)^{-1} z^{1-a / \sigma^{2}}-1\right)}{q(1-x)\left(\pi_{0}-B z\right)} & \text { if } z \in\left[z_{1}, z_{2}\right] \\ 0 & \text { otherwise. }\end{cases}
$$

Continuity of $\pi_{1}(z)$ implies that it must be that

$$
\frac{\pi_{0} \chi z_{i}^{a / \sigma^{2}}}{\pi_{0} \chi z_{i}^{a / \sigma^{2}}+1-\pi_{0}}=B z_{i}, \quad i=1,2
$$

or

$$
1=B z_{i}+B\left(1-\pi_{0}\right)\left(\pi_{0} \chi\right)^{-1} z_{i}^{1-a / \sigma^{2}}, \quad i=1,2
$$

Notice that because $a>\sigma^{2}$, equation (A29) can have at most two solutions.

We are left with two free parameters: $q$ and $x$. They are determined in equilibrium from (i) requiring $\varphi(z)$ to be a density and (ii) requiring the gamble to have expected return equal to one:

$$
\begin{aligned}
\int_{0}^{\infty} \varphi(z) d z & =1 \\
(1-x) \int_{0}^{\infty} z \varphi(z) d z & =1 .
\end{aligned}
$$

Proof of Proposition 5: First, suppose that the manager is skilled so that the excess return on the alpha-generating technology is $a$. Whenever $q_{t}<1$ the manager's continuation utility is a martingale and therefore solves

$$
0=-r W+W_{q} q_{t}(r+a)+\frac{1}{2} W_{q q} \sigma^{2} q_{t}^{2}
$$

The ODE (A32) has a general solution of the form

$$
W\left(q_{t}\right)=C_{+} q_{t}^{\rho^{+}}+C_{-} q_{t}^{\rho^{-}}
$$


where the constants $\rho^{+}$and $\rho^{-}$solve the quadratic equation:

$$
-r+\rho\left(r+a-\frac{1}{2} \sigma^{2}\right)+\frac{1}{2} \sigma^{2} \rho^{2}=0 .
$$

Solving (A33), we have

$$
\rho^{ \pm}=\frac{-\left(r+a-\frac{1}{2} \sigma^{2}\right) \pm \sqrt{\left(r+a-\frac{1}{2} \sigma^{2}\right)^{2}+2 r \sigma^{2}}}{\sigma^{2}} .
$$

There are two boundary conditions (at 0 and at $q=1$ ):

$$
\begin{aligned}
\lim _{q_{t} \rightarrow 0} W\left(q_{t}\right) & =0, \\
W^{\prime}(1) & =k .
\end{aligned}
$$

Thus,

$$
W\left(q_{t}\right)=k \rho^{-1} q_{t}^{\rho}, \quad \rho=\frac{-\left(r+a-\frac{1}{2} \sigma^{2}\right)+\sqrt{\left(r+a-\frac{1}{2} \sigma^{2}\right)^{2}+2 r \sigma^{2}}}{\sigma^{2}} .
$$

If the manager is unskilled then $a=0$ and $\rho=1$. Thus if the manager is skilled with probability $\pi_{t}$ then her continuation utility is

$$
W\left(q_{t}\right)=k\left(\pi_{t} \rho^{-1} q_{t}^{\rho}+\left(1-\pi_{t}\right) q_{t}\right) .
$$

Q.E.D.

Proof of Proposition 6: First, we compute the expected surplus that the manager expects to get from new investors if she reopens her fund when its size is $q^{*}$. As in Proposition 5, we first consider the case in which the manager is skilled with probability one. Let $x_{t}=\ln \left(q_{t}^{o} / q^{*}\right)$. Then

$$
d x_{t}=\left(r+a-\frac{1}{2} \sigma^{2}\right) d t+\sigma d B_{t}, \quad x_{0}=0 .
$$


Let $m_{t}$ be the running maximum of $x_{t}, m_{t}=\max _{0 \leq s \leq t} x_{t}$. The manager is paid whenever $x_{t}=m_{t}$. Whenever $x_{t}<m_{t}$ the manager's continuation utility follows a martingale and therefore solves

$$
0=-r W^{n}+W_{x}^{n}\left(r+a-\frac{1}{2} \sigma^{2}\right)+\frac{1}{2} W_{x x}^{n} \sigma^{2}
$$

The ODE (A38) has a general solution of the form

$$
W^{n}\left(x_{t}, m_{t}\right)=f\left(m_{t}\right) e^{\rho^{+} x_{t}}+g\left(m_{t}\right) e^{\rho^{-} x_{t}}
$$

where the constants $\rho^{+}$and $\rho^{-}$solve the quadratic equation (A33), and therefore are as in (A34). $f\left(m_{t}\right)$ and $g\left(m_{t}\right)$ are arbitrary functions of $m_{t}$. Because $\lim _{x_{t} \rightarrow-\infty} W^{n}\left(x_{t}\right)=0$ it must be that $g\left(m_{t}\right) \equiv 0$. Without loss of generality, the solution is

$$
W^{n}\left(x_{t}, m_{t}\right)=e^{\rho\left(x_{t}-m_{t}\right)} f\left(m_{t}\right), \quad \rho=\rho^{+} .
$$

The boundary condition at $x_{t}=m_{t}$ is

$$
f^{\prime}\left(m_{t}\right)-\rho f\left(m_{t}\right)+k\left(1-q^{*} e^{m_{t}}\right)=0 .
$$

A general solution to (A39) is

$$
f\left(m_{t}\right)=k\left(\frac{q e^{m_{t}}}{1-\rho}+\frac{1}{\rho}\right)+C e^{\rho m_{t}}
$$

where $C$ is a constant. When $q_{t}^{o}$ reaches 1 for the first time, the stake of the new investors in the fund becomes zero. It implies that $f\left(-\ln \left(q^{*}\right)\right)=0$, and therefore

$$
W^{n}\left(x_{t}^{o}, h_{t}\right)=k\left(\frac{x_{t}^{o}}{h_{t}}\right)^{\rho}\left(\frac{1-h_{t}^{\rho}}{\rho}+\frac{h_{t}-h_{t}^{\rho}}{1-\rho}\right)
$$


where $h_{t}=q^{*} e^{m_{t}}$. Thus

$$
W^{n}\left(q^{*}\right) \equiv W^{n}\left(q^{*}, q^{*}\right)=k\left(\frac{1-\left(q^{*}\right)^{\rho}}{\rho}+\frac{q^{*}-\left(q^{*}\right)^{\rho}}{1-\rho}\right) .
$$

Cox and Miller (1965) show that

$$
E e^{-r \nu_{q^{*}}}=\frac{\rho-\rho^{-}}{\rho\left(q^{*}\right)^{\rho^{-}}-\rho^{-}\left(q^{*}\right)^{\rho}} .
$$

Thus, the present value of the opportunity to reopen the fund at date 0 if the manager uses only her alpha-generating technology is

$$
V=\sup _{q^{*}} k \frac{\rho-\rho^{-}}{\rho\left(q^{*}\right)^{\rho^{-}}-\rho^{-}\left(q^{*}\right)^{\rho}}\left(\frac{1-\left(q^{*}\right)^{\rho}}{\rho}+\frac{q^{*}-\left(q^{*}\right)^{\rho}}{1-\rho}\right) .
$$

Suppose at time 0, the manager takes a gamble that delivers a gross return $q^{*}<1$ with probability $p$ and a gross return $1+p\left(1-q^{*}\right) /(1-p)$ with probability $(1-p)$. Suppose also that if a return $q^{*}$ is realized then the manager reopens the fund. Then the expected continuation utility of the manager is

$$
k\left[p\left(\frac{q^{*}}{\rho}+W^{n}\left(q^{*}\right)\right)+(1-p)\left(p\left(1-q^{*}\right) /(1-p)+\frac{1}{\rho}+V\right)\right] .
$$

The manager will gamble if and only if the above utility is greater than her expected utility if she does not gamble. Thus she will gamble if and only if

$$
\sup _{q^{*}} W^{n}\left(q^{*}\right)-\frac{\left(1-q^{*}\right)(1-\rho)}{\rho}>V
$$

Direct computations show that

$$
\sup _{q^{*}} W^{n}\left(q^{*}\right)-\frac{\left(1-q^{*}\right)(1-\rho)}{\rho}=1 .
$$

The supremum is achieved at $q^{*}=0$. So the skilled manager will gamble if $V<1$. By 
looking at $\rho$ and $\rho^{-}$one can see that they depend only on $r / \sigma^{2}$ and $a / \sigma^{2}$. Direct but tedious calculations show that $V<1$ as long as $r / \sigma^{2}>0.1$.

Q.E.D.

Proof of Lemma 5: The integrand in (29) is first convex and then concave in $R$. We show in the electronic appendix that this implies that the optimal gamble is a simple binary gamble such that for some $R>1$, the manager earns $R$ with probability $1 / R$, and loses everything otherwise. Therefore,

$$
\Pi(\pi, t) \equiv \sup _{R \geq 1} R^{\lambda-1} E_{0}\left[h\left(\pi_{t}, R\right) \mid \pi_{0}=\pi\right],
$$

where

$$
h(\pi, R)=\frac{\pi}{1-\pi+\pi R^{\lambda}}, \quad \lambda=\frac{a}{\sigma^{2}} .
$$

For any $R \geq 1, h(\pi, R)$ is a concave function of $\pi$, which implies that $h\left(\pi_{t}, R\right)$ is a super-martingale. Therefore, $\Pi(\pi, t)$ is a decreasing function of $t$. As $t \rightarrow \infty$, $E_{0}\left[h\left(\pi_{t}, R\right) \mid \pi_{0}=\pi\right] \rightarrow \pi R^{-\lambda}$. Therefore, for large $t, \Pi(\pi, t) \leq \pi R^{-1}$, and the optimal choice of $R$ is one, that is not to gamble. Direct computations show that if $\pi>\frac{\lambda-1}{\lambda}$ then

$$
\pi \leq \Pi(\pi, t) \leq \sup _{R \geq 1} R^{\lambda-1} h(\pi, R)=\pi
$$

Q.E.D.

LEMMA 6: Suppose condition 15 holds. Then there exists a finite limit

$$
0<\lim _{\pi \rightarrow 0} V(\pi) \pi^{-\alpha}<\infty
$$

Proof of Lemma 6: We have

$V(\pi)=\int_{0}^{1} G(\pi, x) x^{\alpha} d x=\frac{2 \sigma^{2}}{a^{2} \psi}\left[\begin{array}{c}(1-\pi)^{\frac{1}{2}(1+\psi)} \pi^{\frac{1}{2}(1-\psi)} \int_{0}^{\pi}(1-x)^{-\frac{1}{2}(\psi+3)} x^{\alpha+\frac{1}{2}(\psi-3)} d x \\ +(1-\pi)^{\frac{1}{2}(1-\psi)} \pi^{\frac{1}{2}(1+\psi)} \int_{\pi}^{1}(1-x)^{\frac{1}{2}(\psi-3)} x^{\alpha-\frac{1}{2}(3+\psi)} d x\end{array}\right]$, 
where $\psi$ is defined in (8). Further $r \sigma^{2}>\frac{a^{2}}{2} \alpha(\alpha-1)$ implies that $\psi>2 \alpha-1$. Therefore,

$$
\exists \lim _{\pi \rightarrow 0}(1-\pi)^{\frac{1}{2}(1-\psi)} \pi^{\frac{1}{2}(1+\psi)} \int_{\pi}^{1}(1-x)^{\frac{1}{2}(\psi-3)} x^{\alpha-\frac{1}{2}(3+\psi)} d x \times \pi^{-\alpha}<\infty
$$

and

$$
\exists \lim _{\pi \rightarrow 0}(1-\pi)^{\frac{1}{2}(1+\psi)} \pi^{\frac{1}{2}(1-\psi)} \int_{0}^{\pi}(1-x)^{-\frac{1}{2}(\psi+3)} x^{\alpha+\frac{1}{2}(\psi-3)} d x \times \pi^{-\alpha}<\infty .
$$

Q.E.D. 


\section{Internet Appendix for "Rewarding Trading Skills Without Inducing Gambling" Optimal Risk Shifting - The Dual Approach}

I have not edited from here onwards in accordance with your wishes In this Appendix, we solve for the optimal payoff distribution, holding the mean constant, chosen by an agent who seeks to maximize the expected value of a function $U$ over this payoff. Formally, let $M$ denote the set of Borelian probability measures over $[0,+\infty)$. We seek to solve for the following problem:

$$
\begin{aligned}
& \sup _{\mu \in M} \int_{0}^{\infty} U(R) d \mu(R) \\
& \text { s.t. } \quad \int_{0}^{\infty} R d \mu(R)=1 .
\end{aligned}
$$

Given the primal problem (B1), its dual problem takes the following form:

$$
\begin{aligned}
P^{*}(U) & \equiv \inf _{\left(z_{1}, z_{2}\right) \in \mathbb{R}^{2}} z_{1}+z_{2} \\
\text { s.t. } \forall y & \geq 0, z_{1}+y z_{2} \geq U(y),
\end{aligned}
$$

where $z_{1}$ and $z_{2}$ are some real numbers. Let $P(U)$ and $P^{*}(U)$ denote the solution of the primal and dual problems correspondingly. The dual problem minimizes the value at 1 of a straight line that is above the graph of $U$. The next Proposition shows that under the mild restriction (B3) on the utility function $U$, solutions to the primal and dual problems coincide. Thus the dual approach generates a simple and practical determination of $P(U)$. The role of condition (B3) is to ensure that the infimum is

reached in the dual problem (B2). Graphically, it means that the function $U$ does not have an asymptote with a strictly positive slope. 
PROPOSITION B1: Let $U:[0,+\infty) \rightarrow \mathbb{R}$ be a continuous function such that

$$
\lim _{y \rightarrow+\infty} \frac{U(y)}{y}=0
$$

then

$$
P(U)=P^{*}(U)
$$

Proof of Proposition B1: Observe that condition (B3) implies that the set $Z=$ $\left\{\left(z_{1}, z_{2}\right): \forall y \geq 0, z_{1}+y z_{2} \geq U(y)\right\}$ is nonempty, closed, and there exists $K$ such that

$$
\left(z_{1}, z_{2}\right) \in Z \rightarrow z_{1} \geq K, z_{2} \geq K
$$

The function $\left(z_{1}, z_{2}\right) \rightarrow z_{1}+z_{2}$ is continuous. Thus, there exists $\left(z_{1}^{*}, z_{2}^{*}\right) \in Z$ such that $P^{*}(U)=z_{1}+z_{2}$. Condition (B3) readily implies that $z_{2} \geq 0$.

Let $\mu$ be a probability measure that satisfies (B1). Since for any probability measure $\mu$ that satisfies (B1):

$$
z_{1}^{*}+z_{2}^{*}=\int_{0}^{\infty}\left(z_{1}+R z_{2}\right) d \mu(R) \geq \int_{0}^{\infty} U(R) d \mu(R),
$$

it implies that

$$
P^{*}(U) \geq P(U)
$$

Let us show that the reverse inequality also holds. Without loss of generality, we assume that $U$ has a compact support (for all $U$ satisfying (B3), there clearly exists a continuous function $V$ that has a compact support and such that $V \leq U$ and $\left.P^{*}(V)=P^{*}(U)\right)$. It is straightforward to see that

1. $P^{*}\left(U_{1}\right) \leq P^{*}\left(U_{2}\right)$ for $U_{1}, U_{2}$ such that $U_{1} \leq U_{2}$,

2. $P^{*}(\lambda U)=\lambda P^{*}(U)$ for $U$ and $\lambda \in[0,+\infty)$,

3. $P^{*}\left(U_{1}+U_{2}\right) \leq P^{*}\left(U_{1}\right)+P^{*}\left(U_{2}\right)$ for $U_{1}, U_{2}$. 
Thus, $P^{*}(\cdot)$ is a positively homogeneous and subadditive functional. The HahnBanach Theorem therefore implies that for any $U$ there exists a positive linear functional $L_{U}$, defined on continuous functions with a compact support, such that $L_{U} \leq P^{*}$ and $L_{U}(U)=P^{*}(U)$. By the Riesz representation Theorem, there exists a Borelian measure $\mu_{U}$ on $[0, \infty)$ such that for all $V$

$$
L_{U}(V)=\int_{0}^{\infty} V(R) d \mu_{U}(R) .
$$

For $M>1$, let $u_{M}, v_{M}$ be continuous functions with a compact support such that

$$
\begin{aligned}
& u_{M}(x)=1 \text { on }[0, M], x \geq M \rightarrow u_{M} \leq 1, \\
& v_{M}(x)=x \text { on }[0, M], x \geq M \rightarrow v_{M} \leq M .
\end{aligned}
$$

Clearly,

$$
P^{*}\left(u_{M}\right)=P^{*}\left(v_{M}\right)=1
$$

Then

$$
\begin{aligned}
& L_{U}\left(u_{M}\right)=\int_{0}^{\infty} u_{M}(R) d \mu_{U}(R) \leq P^{*}\left(u_{M}\right)=1, \\
& L_{U}\left(v_{M}\right)=\int_{0}^{\infty} v_{M}(R) d \mu_{U}(R) \leq P^{*}\left(v_{M}\right)=1 .
\end{aligned}
$$

Letting $M \rightarrow+\infty$ implies

$$
\int_{0}^{\infty} d \mu_{U}(R) \leq 1, \int_{0}^{\infty} R d \mu_{U}(R) \leq 1,
$$

and thus

$$
P^{*}(U)=L_{U}(U)=\int_{0}^{\infty} U(R) d \mu_{U}(R) \leq P(U) .
$$

Q.E.D.

PROPOSITION B2: Assume $U$ satisfies (B3), and is continuous and increasing. If the 
solution to the problem $(B 1)$ is non-degenerate $(\mu(1) \neq 1)$ then $P(U)$ can be attained with a binary payoff. Moreover, if $U$ is twice differentiable and has at most one inflexion point, then a necessary and sufficient condition for the solution to (B1) to be non-degenerate is that $U^{\prime}(1)>U(1)-U(0)$. The binary payoff in this case is of the form $\{0, R\}$, where $R>1$.

Proof of Proposition B2: Let a continuous increasing function $U$ satisfy condition (B3). Let $\left(z_{1}, z_{2}\right) \in \mathbb{R}^{2}$ be the solution to the dual problem (B2) associated with $U$. Clearly, $z_{2}>0$. Let

$$
S=\left\{y \geq 0: z_{1}+z_{2} y=U(y)\right\}
$$

Condition B3 and continuity of $U$ imply that $S$ is a nonempty compact set. Let

$$
y_{1}=\min S, \quad y_{2}=\max S
$$

We now proceed in two steps.

Step 1. First, we show that $y_{1} \leq 1 \leq y_{2}$. We prove that $y_{2} \geq 1$. The proof that $y_{1} \leq 1$ is symmetric. Suppose the opposite that $y_{2}<1$ then for some $\varepsilon \in\left(0,1-y_{2}\right)$, let

$$
\eta(\varepsilon)=\min _{y \geq y_{2}+\varepsilon}\left\{\frac{z_{1}-U(y)}{y}+z_{2}\right\} .
$$

Condition B3 and continuity of $U$ imply that $\eta(\varepsilon)>0$.

Define $\left(z_{1}^{\prime}, z_{2}^{\prime}\right)$ as $z_{1}^{\prime}=z_{1}+\left(y_{2}+\varepsilon\right) \eta(\varepsilon), z_{2}^{\prime}=z_{2}-\eta(\varepsilon)$. The pair $\left(z_{1}^{\prime}, z_{2}^{\prime}\right)$ satisfies (B2). To see this, notice that $z_{1}^{\prime}+y z_{2}^{\prime}=z_{1}+y z_{2}+\eta(\varepsilon)\left(y_{2}+\varepsilon-y\right)$. Thus $z_{1}^{\prime}+y z_{2}^{\prime}>$ $z_{1}+y z_{2} \geq U(y)$ for $y<y_{2}+\varepsilon$. Further, $z_{1}^{\prime}+y z_{2}^{\prime} \geq z_{1}+y z_{2}-\eta(\varepsilon) y \geq U(y)$ for $y \geq y_{2}+\varepsilon$ by definition of $\eta(\varepsilon)$. At the same time,

$$
z_{1}^{\prime}+z_{2}^{\prime}=z_{1}+z_{2}+\left(y_{2}+\varepsilon-1\right) \eta(\varepsilon)<z_{1}+z_{2}
$$

which contradicts the definition of $\left(z_{1}, z_{2}\right)$. Thus it must be that $y_{2} \geq 1$. 
Step 2. If $y_{1}=y_{2}$, then Step 1 implies that $S=\{1\}$, and the gamble is degenerate. If $y_{1}<y_{2}$, we have

$$
\begin{aligned}
& z_{1}+y_{1} z_{2}=U\left(y_{1}\right), \\
& z_{1}+y_{2} z_{2}=U\left(y_{2}\right),
\end{aligned}
$$

so that

$$
z_{1}+z_{2}=\frac{1-y_{1}}{y_{2}-y_{1}} U\left(y_{2}\right)+\frac{y_{2}-1}{y_{2}-y_{1}} U\left(y_{1}\right)
$$

From $(\mathrm{B} 4), P(U)=P^{*}(U)$ is attained with a payoff equal to $y_{1}$ with probability $\frac{y_{2}-1}{y_{2}-y_{1}}$ and $y_{2}$ with probability $\frac{1-y_{1}}{y_{2}-y_{1}}$.

We now prove the last part of the proposition. If $U$ has at most one inflexion point, condition (B3) implies that $U$ is either concave - in which case the gamble is degenerate - or convex then concave. Consider the latter case. If the solution is degenerate then $P^{*}(U)$ must be solved by the tangent to $U$ at 1 , which requires $U^{\prime}(1) \leq U(1)-U(0)$. Suppose now that $U^{\prime}(1) \leq U(1)-U(0)$. It implies that $U$ is concave over $[1,+\infty)$ (because $\frac{U(y)-U(0)}{y}$ is decreasing in $y=1$ ). In this case, the tangent to $U$ at 1 solves $P^{*}(U)$, so the gamble is degenerate. Finally, if the solution is nondegenerate then it is attained with a tangent that starts at 0 .

Q.E.D. 


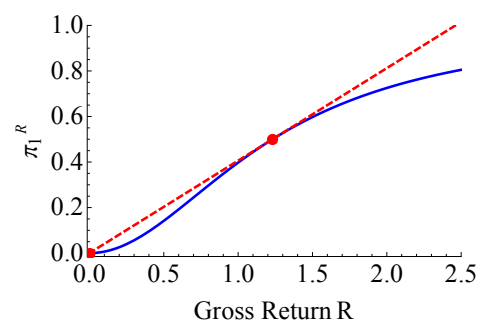

Panel A

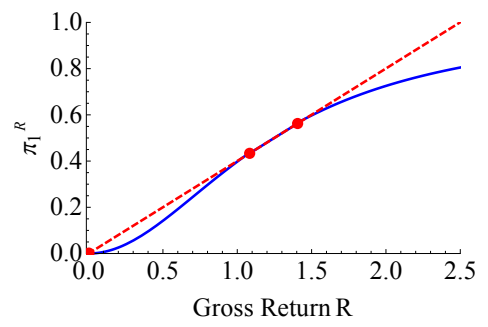

Panel B

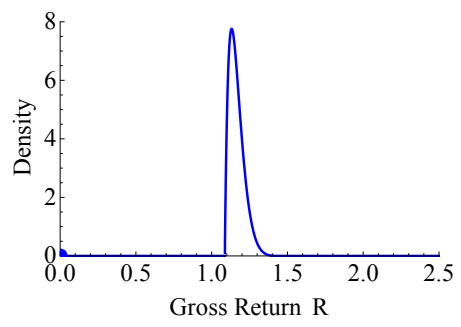

Panel C

Figure 1. Taking on hidden tail risk. Figure 1 illustrates an example considered in Section $C$.

Parameter values are as follows: $\sigma=10 \%, a=2 \sigma^{2}=2 \%, \pi_{0}=40 \%$, and $\gamma=10 \%$. Panel (a), solid blue line, shows a reputation of the manager at date 1 as a function of the realized gross return $R$ when investors believe that the manager does not gamble. The optimal gamble delivers either 0 or a gross return of 1.23 with probability of $80 \%$. Panel (b), solid blue line, displays a reputation of the manager in the equilibrium in which investors rationally take into account that the manager gambles. In equilibrium, the manager gambles with probability $q=5.6 \%$. When she gambles, the manager loses everything with probability $x=8.8 \%$ and obtains a gross return between 1.02 and 1.49 . Panel (c) shows the gamble's density. 